\title{
One-Sided Invertibility of Toeplitz Operators on the Space of Real Analytic Functions on the Real Line
}

\author{
M. Jasiczak@ and A. Golińska
}

\begin{abstract}
We show that a Toeplitz operator on the space of real analytic functions on the real line is left invertible if and only if it is an injective Fredholm operator, it is right invertible if and only if it is a surjective Fredholm operator. The characterizations are given in terms of the winding number of the symbol of the operator. Our results imply that the range of a Toeplitz operator (and also its adjoint) is complemented if and only if it is of finite codimension. Similarly, the kernel of a Toeplitz operator (and also its adjoint) is complemented if and only if it is of finite dimension.
\end{abstract}

Mathematics Subject Classification. Primary 47B35, 46E10; Secondary 47A05, 30H99, 30H50, 46A13.

Keywords. Toeplitz operator, Real analytic, One-sided invertible, Cauchy transform.

\section{Introduction}

In this paper we study Toeplitz operators on the space of real analytic functions on the real line. We start by presenting the classical background for our investigation. The presentation is based on the beautiful books by Gelfond [21] and Markushevich [34]. In the next section we discuss our main results.

A fundamental result in function theory says that a holomorphic function locally develops into a power series. There are however also other series representations of holomorphic functions. Some holomorphic functions develop into Newton series

$$
a_{0}+\sum_{n=1}^{\infty} \frac{a_{n}}{n !}\left(z-z_{0}\right) \ldots\left(z-z_{n-1}\right),
$$

The research was supported by National Center of Science (Poland), Grant No. UMO2013/10/A/ST1/00091. 
where $\left(z_{n}\right)$ is an appropriately chosen sequence of complex numbers. Such representations are deeply connected with interpolation theory and difference equations (actually the whole Gelfond's book [21] is devoted to the study of this relation). We briefly recall now the construction of Newton interpolation polynomials in order to provide motivation for our study. This leads to representations of form (1). Let $z_{0}, \ldots, z_{n} \in \mathbb{C}$ be pairwise different. A function $f: \mathbb{C} \rightarrow \mathbb{C}$ can be written in the following way:

$$
\begin{aligned}
f(z)= & {\left[z_{0}\right]+\left[z_{0}, z_{1}\right]\left(z-z_{0}\right)+\left[z_{0}, z_{1}, z_{2}\right]\left(z-z_{0}\right)\left(z-z_{1}\right)+\cdots } \\
& +\left[z_{0}, \ldots, z_{n}\right]\left(z-z_{0}\right) \ldots\left(z-z_{n-1}\right) \\
& +\left[z, z_{0}, \ldots, z_{n}\right]\left(z-z_{0}\right) \ldots\left(z-z_{n}\right) \\
:= & P_{n}(z)+R_{n}(z)
\end{aligned}
$$

where

$$
R_{n}(z):=\left[z, z_{0}, \ldots, z_{n}\right]\left(z-z_{0}\right) \ldots\left(z-z_{n}\right) .
$$

Here $\left[z_{0}\right]=f\left(z_{0}\right)$ and $\left[z_{0}, \ldots, z_{k}\right], k=1, \ldots, n$ are the divided differences

$$
\begin{aligned}
{\left[z_{0}, z_{1}\right] } & :=\frac{\left[z_{0}\right]-\left[z_{1}\right]}{z_{0}-z_{1}}=\frac{f\left(z_{0}\right)-f\left(z_{1}\right)}{z_{0}-z_{1}} \\
\ldots & \\
{\left[z_{0}, \ldots, z_{n}\right]: } & =\frac{\left[z_{0}, z_{1}, \ldots, z_{n-1}\right]-\left[z_{1}, \ldots, z_{n}\right]}{z_{0}-z_{n}} .
\end{aligned}
$$

One easily notices that $P_{n}$ is a polynomial such that $P_{n}\left(z_{k}\right)=f\left(z_{k}\right)$ for $k=0, \ldots, n$. This polynomial is called a Newton interpolation polynomial (actually it is rather the Newton form of interpolation polynomial). It is worth mentioning that it appears for the first time in Newton's Principia Mathematica (Book III, Lemma V, Case 1).

Given a function $f$ and a sequence $\left(z_{n}\right)$, a natural question is whether the polynomials $P_{n}$ converge yielding representation of the form (1) with

$$
\frac{a_{n}}{n !}=\left[z_{0}, \ldots, z_{n-1}\right]
$$

One immediately notices a similarity between Newton series and Taylor series. In fact, the series (1) with coefficients defined by divided differences (3) can be considered as a discrete analog of Taylor series. The divided differences play the role of discrete analogs of the consecutive derivatives, the polynomials $\left(z-z_{0}\right) \ldots\left(z-z_{n-1}\right)$ of the functions $z^{n}$. The question, which we formulated above, that is, whether the polynomials $P_{n}$ converge is deep and does not have an easy answer. Some answers are known for entire functions (see [21], Satz 1, p. 43, Satz 1, p. 47 and also Chapter II, $\S 2$ and $\S 3)$.

Crucial for our study is the fact that the operators connected with Newton series turn out to be (appropriately defined) Toeplitz operators, the object of our investigation. Namely, it follows easily from the residue theorem for holomorphic functions that

$$
\left[z, z_{0}, \ldots, z_{n}\right]=\frac{1}{2 \pi i} \int_{\gamma} \frac{f(\zeta)}{\prod_{k=0}^{n}\left(\zeta-z_{k}\right)} \frac{d \zeta}{\zeta-z},
$$


when $f$ is (for simplicity) entire and $\gamma$ is a $C^{\infty}$ smooth Jordan curve such that the points $z, z_{0}, \ldots, z_{n}$ are contained in $I(\gamma)$, the interior of the curve $\gamma$ (recall Jordan's theorem). This implies that the rest term $R_{n}$ in (2) is, as we shall explain shortly, a composition of Toeplitz operators. Let us reformulate this observation: convergence properties of Newton series are governed by Toeplitz operators. Also, divided differences $\left[z_{0}, \ldots, z_{k}\right]$, which are the coefficient functionals in (1), are just the evaluations of the value of a Toeplitz operator at one of the points $z_{0}, \ldots, z_{k}$.

An important situation occurs when $z_{k}=k, k=0, \ldots, n$. Then

$$
\left[z_{0}, \ldots, z_{n}\right]=\frac{(-1)^{n}}{n !} D_{n}[f]=\frac{(-1)^{n}}{n !} \sum_{k=0}^{n}\left(\begin{array}{l}
n \\
k
\end{array}\right)(-1)^{k} f(k)
$$

and the corresponding Cauchy integral (4) is usually called Nörlund-Rice integral. The method of estimating it is considered as 'one of the basic asymptotic techniques of the analysis of algorithms'. We refer the reader to [19] and [38] for an enlightening discussion of the applications to theoretical computer science and discrete mathematics.

Observe that if $f$ can be represented by Newton series (1) then $f\left(z_{k}\right)$ is given by a finite sum $a_{0}+\sum_{n=1}^{k} \frac{a_{n}}{n !}\left(z_{k}-z_{0}\right) \ldots\left(z_{k}-z_{k-1}\right)$. This property was used by Pólya to prove his result which characterizes functions holomorphic in some half-space $\Re z>\alpha$, satisfying some growth condition which vanish at integers, as polynomials ([21], p. 133, Satz 10). Even more importantly, the fact that the function $e^{\alpha z}$ develops into a Newton series is one of the key elements in the proof of the theorem which says that $\alpha$ and $e^{\alpha}$ cannot be simultaneously algebraic numbers ([21], p. 167, Satz 9). This in particular implies that the numbers $e$ and $\pi$ are transcendental. This result contains as a special case Lindemann classical theorem. We mention that Newton series representations draw some attention also in combinatorics in the so called umbral calculus [41]. There is an ongoing interest in Dirichlet series. Therefore it is worthy of mentioning that there is a relation between Newton series and Dirichlet series (see [21], p. 113, Satz 3).

Roughly speaking a divided difference considered as a function of one its arguments

$$
z \mapsto\left[z, z_{0}, \ldots, z_{n}\right]
$$

is well-defined and formula (4) holds true for functions which develop into power series. This is why we consider in this paper the case of real analytic functions. Recall that these are the functions $f: \mathbb{R} \rightarrow \mathbb{C}$ which develop locally, around each point of $\mathbb{R}$ into convergent power series. This means that a real analytic function on $\mathbb{R}$ is a germ on $\mathbb{R}$ of a holomorphic function. To sum this up, we study in this paper a class of operators, which includes the operators defined by divided differences (4), on functions which locally develop into power series. Observe that the assumption that $f$ is real analytic is precisely what is needed to consider Nörlund-Rice formulae (4) and (5).

The space of all real analytic functions on $\mathbb{R}$ will be denoted by $\mathcal{A}(\mathbb{R})$. This space is not a Banach space, it is not even metrizable. It carries however a natural locally convex topology and such fundamental tools of functional 
analysis as the Hahn-Banach theorem, the uniform boundedness principle and the open mapping/closed graph theorem are available. This will play some role in our proofs.

Somewhat imprecise one may say that we consider here the space of all holomorphic functions on which we impose only one condition: their domain must contain the real line. One should compare this for instance with the Hardy, Bergman or Fock spaces in which cases the domain of a holomorphic function is fixed and common for all elements in the space. There are excellent books concerning Toeplitz operators on these spaces [4,37,47]. Real analytic functions play a significant role in mathematics. It suffices to mention here such fundamental results as the Cauchy-Kovalevskaya Theorem or Holmgren's Uniqueness Theorem (cf. [40], Theorems 2.21 and 2.26), which are formulated for this class of functions. Importantly, Hörmander characterized in [25] partial differential operators with constant coefficients which are surjective on the space of real analytic functions on a domain in $\mathbb{R}^{n}$. In [32] Langenbruch solved the right inverse problem for convolution operators on the space of real analytic functions. In this paper we consider similar problems for the class of Toeplitz operators, which we introduced in [9]. Our approach is however completely different.

A Toeplitz operator $T_{F}$ on $\mathcal{A}(\mathbb{R})$ is an operator of the form

$$
T_{F}:=\mathcal{C} M_{F} .
$$

The symbol $F$ of the operator $T_{F}$ belongs to the symbol space $\mathcal{X}(\mathbb{R})$, which is defined as the inductive limit of the spaces $H(U \backslash K)$ - the spaces of all functions holomorphic in $U \backslash K$,

$$
\mathcal{X}(\mathbb{R}):=\operatorname{limind} H(U \backslash K) .
$$

Here the sets $U$ run through complex neighborhoods of $\mathbb{R}$ and the sets $K$ run through compact subsets of $\mathbb{R}$.

If $U$ is an open neighborhood of $\mathbb{R}, K$ is a compact subset of $\mathbb{R}$ and $\tilde{F}$ is holomorphic in $U \backslash K$, then $\tilde{F}$ determines an element in $\mathcal{X}(\mathbb{R})$, which is the equivalence class $[\tilde{F}]_{\sim}$ in limind $H(U \backslash K)$ of the function $\tilde{F}$. Two such functions $\tilde{F}_{i} \in H\left(U_{i} \backslash K_{i}\right), i=1,2$ determine the same element if for some open $U \subset U_{1} \cap U_{2}$ containing $\mathbb{R}$ and compact $K \supset K_{1} \cup K_{2}$ contained in $\mathbb{R}$ it holds that

$$
\left.\tilde{F}_{1}\right|_{U \backslash K}=\left.\tilde{F}_{2}\right|_{U \backslash K}
$$

In (6) the operator $M_{F}: \mathcal{X}(\mathbb{R}) \rightarrow \mathcal{X}(\mathbb{R})$ is the operator of multiplication by $F$ and $\mathcal{C}: \mathcal{X}(\mathbb{R}) \rightarrow \mathcal{A}(\mathbb{R})$ is the Cauchy transform. We remark here that $\mathcal{C}$ turns out be a projection onto the space of real analytic functions $\mathcal{A}(\mathbb{R})$. We recall the details in Sect. 3 .

Observe that if $z_{0}, \ldots, z_{n}$ are real then the operator given by (4) is precisely an operator $T_{F}$ with $F$ which is the equivalence class in $\mathcal{X}(\mathbb{R})$ of the function

$$
\frac{1}{\prod_{k=0}^{n}\left(\zeta-z_{k}\right)},
$$

which belongs to $H\left(\mathbb{C} \backslash\left\{z_{0}, \ldots, z_{n}\right\}\right)$. 
A fundamental result of Brown and Halmos [5] says that a Toeplitz operator on the Hardy space $H^{2}(\mathbb{T})$ is of the form $P M_{\phi}$, where $M_{\phi}$ is the operator of multiplication by $\phi \in L^{\infty}(\mathbb{T})$ and $P: L^{2}(\mathbb{T}) \rightarrow H^{2}(\mathbb{T})$ is the Riesz projections (which is also the Cauchy transform in the unit disk). Observe a similarity between this characterization of Toeplitz operators and formula (6). The similarity is deeper and it motivated our research. We mention here that in ([9], Theorem 2) we proved a characterization of Fredholm Toeplitz operators on $\mathcal{A}(\mathbb{R})$ which strongly resembles the classical result for Hardy spaces. We shall recall this result in the next section, since it is one of the main tools in this paper.

Toeplitz operators on $H^{2}(\mathbb{T})$ are defined by a well-known matrix condition. Such a characterization holds true also in the case of the space of real analytic functions $\mathcal{A}(\mathbb{R})$. Any operator of the form $T_{F}, F \in \mathcal{X}(\mathbb{R})$ satisfies

$$
T_{F}\left(x^{n}\right)(\xi)=a_{-n}+a_{-n+1} \xi+a_{-n+2} \xi^{2}+\cdots
$$

locally near zero for complex numbers

$$
a_{n}=\frac{1}{2 \pi i} \int_{\gamma} \tilde{F}(\zeta) \zeta^{-n-1} d \zeta .
$$

Here, $\tilde{F} \in H(U \backslash K)$ is a representative of $F \in \mathcal{X}(\mathbb{R})$. The set $U$ is an open simply connected neighborhood of $\mathbb{R}$ and $K$ is a compact subset of $\mathbb{R}$. The symbol $\gamma$ stands for (any) $C^{\infty}$ smooth Jordan curve in $U \backslash K$ which contains the origin and the set $K$ in its interior $I(\gamma)$.

Condition (7) says that if we consider Taylor series expansions around zero of the real analytic functions $T_{F}\left(x^{n}\right), n \in \mathbb{N}_{0}$ and create an infinite matrix $M_{T_{F}}$ by putting the Taylor coefficients of the functions $T_{F}\left(x^{n}\right)$ in the consecutive columns of the matrix $M_{T_{F}}$, then this matrix is an (infinite) Toeplitz matrix

$$
M_{T_{F}}=\left(\begin{array}{cccc}
a_{0} & a_{-1} & a_{-2} & \ldots \\
a_{1} & a_{0} & a_{-1} & \ldots \\
a_{2} & a_{1} & a_{0} & \ldots \\
\ldots & \ldots & \ldots & \ddots
\end{array}\right) .
$$

Naturally such a matrix $M_{T}$ can be created for any operator $T: \mathcal{A}(\mathbb{R}) \rightarrow$ $\mathcal{A}(\mathbb{R})$, not only for the Toeplitz operators $T_{F}$. We say that the matrix $M_{T}$ is associated with the operator $T$. It was proved in $([9]$, Theorem 1$)$ that if the matrix associated with a continuous linear operator $T: \mathcal{A}(\mathbb{R}) \rightarrow \mathcal{A}(\mathbb{R})$ is a Toeplitz matrix, then $T$ is a Toeplitz operator. That is, $T$ is necessarily an operator $T_{F}$ defined in (6) for some symbol $F \in \mathcal{X}(\mathbb{R})$. This is an analog of the classical Brown-Halmos result.

We emphasize that one should be careful with the matrix interpretation of a Toeplitz operator since monomials do not form a Schauder basis in $\mathcal{A}(\mathbb{R})$. In fact, as shown by Domański and Vogt [17] the space $\mathcal{A}(\mathbb{R})$ has no Schauder basis at all. Note however that condition (7) determines the operator, since polynomials are dense in $\mathcal{A}(\mathbb{R})$.

Our primary motivation in this project has been to build a theory of continuous linear operators on the space $\mathcal{A}(\mathbb{R})$ in a way similar to the known 
theories of operators on Hilbert and Banach spaces. There is an extensive literature on linear continuous operators on these spaces. Much less is known about both concrete and abstract operators on locally convex spaces. There are however prominent exceptions such as differential or convolution operators. Nonetheless the theory of linear continuous operators on locally convex spaces is less developed than the Hilbert or Banach space counterpart. The basic object in our considerations is a matrix associated with an operator. The idea to consider operators determined by matrices associated with them comes from the work of Domański and Langenbruch. In a series of papers [10-13] they created the theory of the so-called Hadamard multipliers. These are the operators the associated matrix of which is just diagonal. This research was continued by Domański et al. [15] and also by Vogt in [44,45] for spaces of distributions, in [43] for spaces of smooth functions and by Trybuła in [42] for spaces of holomorphic functions. In [14] and [16] Domański and Langenbruch showed that this theory provides the correct framework to study Euler's equation. This equation on temperate distributions was studied in [46] by Vogt. Golińska in [23] and [24] studies operators defined by Hankel matrices.

The paper is divided into four sections. In the next one we present our main results. Section three provides background for our study. We recall there basic facts concerning real analytic functions and Toeplitz operators. In that section we also prove an analog of Wiener-Hopf factorization. In section four we prove our main results. This is divided into two subsections. First we discuss left invertibility of Toeplitz operators. The second subsection is devoted to right invertibility of Toeplitz operators.

\section{Main Results}

We shall say that $F \in \mathcal{X}(\mathbb{R})$ does not vanish if $F$ is represented by $\tilde{F} \in$ $H(U \backslash K)$, which does not vanish in $U \backslash K$.

Our main results are the following theorems:

Theorem 1. A Toeplitz operator $T_{F}: \mathcal{A}(\mathbb{R}) \rightarrow \mathcal{A}(\mathbb{R}), F \in \mathcal{X}(\mathbb{R})$, is left invertible if and only if $T_{F}$ is an injective Fredholm operator. Equivalently, $T_{F}$ is left invertible if and only if $F \in \mathcal{X}(\mathbb{R})$ does not vanish and

$$
\text { winding } F \geq 0 \text {. }
$$

Theorem 2. A Toeplitz operator $T_{F}: \mathcal{A}(\mathbb{R}) \rightarrow \mathcal{A}(\mathbb{R}), F \in \mathcal{X}(\mathbb{R})$, is right invertible if and only if $T_{F}$ is a surjective Fredholm operator. Equivalently, $T_{F}$ is right invertible if and only if $F \in \mathcal{X}(\mathbb{R})$ does not vanish and

$$
\text { winding } F \leq 0 \text {. }
$$

For $F \in \mathcal{X}(\mathbb{R})$ the winding number is defined by means of any representative of $F$ which does not vanish. That is, assume that $F=[\tilde{F}] \sim$ for some $\tilde{F} \in H(U \backslash K)$, which does vanish in $U \backslash K$. Here, $U$ is an open neighborhood 
of $\mathbb{R}$ and $K$ is a compact subset of $\mathbb{R}$. We may assume that $U$ is simply connected. Then

$$
\text { winding } F:=\text { winding } \tilde{F}=\operatorname{Ind}_{\tilde{F} \circ \gamma}(0)=\frac{1}{2 \pi i} \int_{\gamma} \frac{\tilde{F}^{\prime}(\zeta)}{\tilde{F}(\zeta)} d \zeta \text {. }
$$

The symbol $\gamma$ stands for a $C^{\infty}$ smooth Jordan curve in $U \backslash K$ such that $K$ is contained in the interior $I(\gamma)$ of the curve $\gamma$. One easily observes that the definition is correct. This follows from Cauchy's theorem.

A crucial tool in the proofs of our main results is the following theorem:

Theorem 2.1. ([9], Theorem 2) A Toeplitz operator $T_{F}, F \in \mathcal{X}(\mathbb{R})$ is a Fredholm operator if and only if $F$ does not vanish. In this case,

$$
\text { index } T_{F}=- \text { winding } F \text {. }
$$

It is worth mentioning that Theorems 1 and 2 are closely analogous to the classical Hardy space theory of Toeplitz operators with continuous symbols (see [22], Theorem 7.1 in accordance with [3], Lemma 6.14). We find it rather surprising. The methods are however completely different, since the Banach algebra techniques are not available in our setting.

The proofs of both Theorems 1 and 2 are based on the characterization of semi-Fredholm operators obtained in [28]. In order to present the idea of the proof we recall now these results.

Let $F \in \mathcal{X}(\mathbb{R})$ and let a holomorphic function $\tilde{F} \in H(U \backslash K)$ be its representative: $F=[\tilde{F}]_{\sim}$ (here $K \subset \mathbb{R}$ is compact and $U \supset \mathbb{R}$ is open).

(i) We will say that $F$ has real zeros going to infinity if there are $x_{n} \in \mathbb{R}$ such that $\lim \left|x_{n}\right|=\infty$ and $\tilde{F}\left(x_{n}\right)=0$.

(ii) We will say that $F$ has non-real zeros accumulating at a real point if the function $\tilde{F}$ has zeros $z_{n} \notin \mathbb{R}$ whose limit $\lim z_{n}$ exists and belongs to $\mathbb{R}$.

We observe that these two properties depend only on the germ $F \in \mathcal{X}(\mathbb{R})$ and do not depend on the choice of $K, U$ and the representative $\tilde{F} \in H(U \backslash K)$ of $F$. The set of zeros of a function $\tilde{F}$ in $H(U \backslash K)$ is discrete in $U \backslash K$. It follows that, given a non-zero germ $F \in \mathcal{X}(\mathbb{R})$, one can find $U, K$ and a representative $\tilde{F} \in H(U \backslash K)$ of $F$ that has no zeros in $U \backslash K$ if and only if $F \in \mathcal{X}(\mathbb{R})$ neither has real zeros going to infinity nor non-real zeros accumulating at a real point.

Theorem 2.2. ([27], Theorem 3) Assume that $F \in \mathcal{X}(\mathbb{R})$ does not vanish identically. Consider the Toeplitz operator $T_{F}$.

(i) If $F$ has no real zeros going to infinity and has no non-real zeros accumulating at a real point, then the kernel of $T_{F}$ is finite dimensional, the cokernel is finite dimensional and the image of $T_{F}$ is closed;

(ii) If $F$ has non-real zeros accumulating at a real point, but has no real zeros going to infinity, then the operator $T_{F}$ is surjective and the kernel of $T_{F}$ is of infinite dimension; 
(iii) If $F$ has real zeros going to infinity, but has no non-real zeros accumulating at a real point, then the operator $T_{F}$ is injective and the range of $T_{F}$ is a closed subspace of infinite codimension;

(iv) If $F$ has both real zeros going to infinity and non-real zeros accumulating at a real point, then the operator $T_{F}$ is injective and the range of $T_{F}$ is a dense subspace of infinite codimension.

For any $F \in \mathcal{X}(\mathbb{R})$ one of the above four cases holds true.

We remark that we may assume that one of the four cases concerning the zeros of $\tilde{F}$ with $[\tilde{F}]_{\sim}=F \in \mathcal{X}(\mathbb{R})$ holds since we may always shrink the open set $U$ and enlarge the compact set $K$ without affecting the equivalence class of $\tilde{F}$ in the symbol space $\mathcal{X}(\mathbb{R})=\operatorname{limind} H(U \backslash K)$ or the operator $T_{F}$.

If condition $(i i)$ of Theorem 2.2 holds then the operator $T_{F}$ is not injective. Hence it cannot be left invertible. Recall that if $S$ is a left inverse of $T_{F}$, $S T_{F}=I$, then $T_{F} \circ S$ is a continuous projection onto the range of $T_{F}$. Thus the range of $T_{F}$ is a closed complemented subspace of $\mathcal{A}(\mathbb{R})$. This implies that under condition $(i v)$ of Theorem 2.2 the operator $T_{F}$ cannot be left invertible. The next result, which is essentially our first main result, deals with condition $($ iii).

Theorem 3. Assume that $F \in \mathcal{X}(\mathbb{R})$ has real zeros going to infinity, but has no non-real zeros accumulating at a real point. The range of the operator $T_{F}$ is not a complemented subspace of $\mathcal{A}(\mathbb{R})$.

Theorem 3 implies that the operator $T_{F}$ can be left invertible only when ( $i$ ) of Theorem 2.2 holds. That is, only when $T_{F}$ is a Fredholm operator. This is the main ingredient of the proof of Theorem 1.

In the proof of Theorem 3 we obtain precise information on the range of the operator $T_{F}$ (see Proposition 4.3). This seems to be interesting per se. We explicitly construct a sequence of functionals $\xi_{n} \in \mathcal{A}(\mathbb{R})^{\prime}$ such that

$$
R\left(T_{F}\right)=\bigcap_{n=1}^{\infty} \operatorname{ker} \xi_{n} .
$$

Then we argue that if the range was complemented, then a natural projection should be in a reasonable way continuous. We show that this is however not the case. The proof is constructive and we show in particular how to obtain by means of Toeplitz operators functions dual to the functionals $\xi_{n}$.

In order to show that an injective Fredholm operator is left invertible one simply uses the closed graph theorem, which, let us emphasize this, works in $\mathcal{A}(\mathbb{R})$. This time there is also a constructive argument. Namely, we complete the picture of Fredholm Toeplitz operators on $\mathcal{A}(\mathbb{R})$ and prove a Wiener-Hopf type factorization in this space (Theorem 3.2 below) actually by mimicking the classical argument. With this tool at hand we may write down a left inverse explicitly.

As we have already remarked, our characterization of one sided invertible Toeplitz operators on $\mathcal{A}(\mathbb{R})$ resembles closely the Hardy space results. There is however a difference. An operator on a Hilbert space fails to be left invertible in two situations: it is not injective or the range is not closed. Note 
that the reason for $T_{F}$ not to be left invertible may be more involved. That is, the range of the operator may not be complemented.

It seems worth mentioning that the study when the range of an operator is complemented drew some attention also in other natural cases [7,29]. By a well-known result of Lindenstrauss and Tzafriri [33] a Banach space is isomorphic to a Hilbert space provided every closed subspace is complemented. Theorems 2.2 and 3 immediately imply the following result:

Corollary 2.3. Consider a Toeplitz operator $T_{F}: \mathcal{A}(\mathbb{R}) \rightarrow \mathcal{A}(\mathbb{R}), F \in \mathcal{X}(\mathbb{R})$. The range of $T_{F}$ is a non-trivial complemented subspace of $\mathcal{A}(\mathbb{R})$ if and only if it is a subspace of non-zero finite codimension. This holds if and only if $F \in \mathcal{X}(\mathbb{R})$ does not vanish and

$$
\text { winding } F>0 \text {. }
$$

The codimension of the range of $T_{F}$ is equal to winding $F$.

Theorem 3 means that there are plenty of closed subspaces of $\mathcal{A}(\mathbb{R})$, which are not complemented. By a trivial argument the subspace of even (odd) real analytic functions is complemented in $\mathcal{A}(\mathbb{R})$. Hence there are complemented subspaces in $\mathcal{A}(\mathbb{R})$ of infinite codimension. This also means that $f(x) \mapsto f(-x)$ is not a Toeplitz operator, which follows immediately from the matrix representation (8) (this operator is however an Hadamard multiplier). Our results provide therefore a method to construct an abundance of subspaces of $\mathcal{A}(\mathbb{R})$ and also $\mathcal{A}(\mathbb{R})_{b}^{\prime}$, which are not complemented.

If an operator $T_{F}: \mathcal{A}(\mathbb{R}) \rightarrow \mathcal{A}(\mathbb{R})$ is right invertible, then it is surjective. It follows therefore from Theorem 2.2 that this happens either if $T_{F}$ is a surjective Fredholm operator or $F=[\tilde{F}]_{\sim}$ for $\tilde{F} \in H(U \backslash K)$, which vanishes only on a sequence $\left(z_{n}\right) \subset \mathbb{C} \backslash \mathbb{R}$, which accumulates only at points of $K$. In the next result, which is essentially our second main result, we rule out the second possibility. Note that if $T_{F}$ is right invertible, then $T_{F}^{\prime}: \mathcal{A}(\mathbb{R})_{b}^{\prime} \rightarrow \mathcal{A}(\mathbb{R})_{b}^{\prime}$ is left invertible and, as a result, its range in $\mathcal{A}(\mathbb{R})_{b}^{\prime}$ is complemented.

Theorem 4. Assume that $F \in \mathcal{X}(\mathbb{R})$ has non-real zeros accumulating at a real point but no real zeros going to infinity. The range of the operator $T_{F}^{\prime}$ is not a complemented subspace of $\mathcal{A}(\mathbb{R})_{b}^{\prime}$.

In order to prove the theorem we again prove a representation of the range of the adjoint operator $T_{F}^{\prime}$ of the form (9).

This theorem implies the following analog of Corollary 2.3:

Corollary 2.4. Consider a Toeplitz operator $T_{F}: \mathcal{A}(\mathbb{R}) \rightarrow \mathcal{A}(\mathbb{R}), F \in \mathcal{X}(\mathbb{R})$. The kernel of $T_{F}$ is a non-trivial complemented subspace of $\mathcal{A}(\mathbb{R})$ if and only if it is a subspace of non-zero finite dimension. This holds if and only if $F \in \mathcal{X}(\mathbb{R})$ does not vanish and

$$
\text { winding } F<0 \text {. }
$$

The dimension of the kernel of $T_{F}$ is equal to - winding $F$. 
The statements concerning the codimension of the range of $T_{F}$ in Corollary 2.3 and the dimension of the kernel of $T_{F}$ in Corollary 2.4 are immediate consequences of the Coburn-Simonenko theorem for Toeplitz operators on $\mathcal{A}(\mathbb{R})$, which we proved in ([28], Theorem 1.2). This says, as in the Hardy space case, that either $\operatorname{ker} T_{F}$ or $\operatorname{ker} T_{F}^{\prime}$ is trivial.

Our work relies heavily on the previous results concerning Fredholm Toeplitz operators $[9,27]$ and semi-Fredholm operators [28]. We also use extensively the Köthe-Grothendieck-da Silva duality. This will be recalled in Sect. 3. A basic tool in our arguments is Weierstrass factorization theory of entire functions. For this we refer the reader to [6].

\section{The Space of Real Analytic Functions and Toeplitz Operators}

\subsection{The Space of Real Analytic Functions}

For the reader's convenience we recall here basic properties of the space of real analytic functions on the real line and define Toeplitz operators on this space. For more details we refer to our previous works [9,27] and, especially, [28]. We refer the reader to $[26,36]$ and [30] for the functional analytic background. Projective and injective limits of locally convex spaces are also studied in detail in [20]. The article [8] gives an instructive introduction to real analytic functions. There is also an interesting book [31], however of less functional analytic flavor. In our presentation of the space $\mathcal{A}(\mathbb{R})$ here we essentially follow [18].

The space of real analytic functions on the real line, denoted $\mathcal{A}(\mathbb{R})$, consists of all functions $f: \mathbb{R} \rightarrow \mathbb{C}$ which develop at every point $x \in \mathbb{R}$ into a power series convergent in a neighborhood of $x$ to $f$. This implies that a real analytic function extends to a holomorphic function on some neighborhood of $\mathbb{R}$ and two such extensions define the same element of $\mathcal{A}(\mathbb{R})$ if they coincide on an open set which contains $\mathbb{R}$. In other words, a function $f \in \mathcal{A}(\mathbb{R})$ is a germ on $\mathbb{R}$ of a holomorphic function and as sets

$$
\mathcal{A}(\mathbb{R})=\operatorname{limind}_{U} H(U),
$$

where the inductive limit runs through all open neighborhoods of $\mathbb{R}$ and $H(U)$ is the Fréchet space of all holomorphic functions on $U$ with the topology of uniform convergence on compact subsets. Equality (10) is used to equip the space $\mathcal{A}(\mathbb{R})$ with the so-called inductive topology. This is the strongest locally convex topology such that every restriction

$$
\left.H(U) \ni f \mapsto f\right|_{\mathbb{R}} \in \mathcal{A}(\mathbb{R})
$$

is continuous.

On the other hand, if $K \subset \mathbb{R}$ is compact then the restriction of $f$ to $K$ determines the germ in $H(K)$, the space of all germs of holomorphic functions over $K$ (we refer the reader to [2], p. 64 for detailed information on this space). Therefore, we may equip $\mathcal{A}(\mathbb{R})$ with the projective topology 
given by

$$
\mathcal{A}(\mathbb{R})=\operatorname{limproj}_{K} H(K),
$$

where the sets $K$ run through all compact subsets of $\mathbb{R}$. One easily observes that instead of taking in (11) all compact subsets $K \subset \mathbb{R}$ one can take any compact exhaustion $\left\{K_{n}\right\}_{n \in \mathbb{N}}$ of $\mathbb{R}$, for example $\{[-n, n]\}_{n \in \mathbb{N}}$.

A fundamental result of Martineau [35] says that the inductive and the projective topology coincide. In [18] the authors give a simpler proof of this fact. Other proofs are discussed in [8]. With this topology the space $\mathcal{A}(\mathbb{R})$ is nuclear, complete and the polynomials are dense. It is also ultrabornological and webbed (see [36], Chapter 24 for definitions). This implies that the open mapping and the closed graph theorems hold for linear maps $T: \mathcal{A}(\mathbb{R}) \rightarrow$ $\mathcal{A}(\mathbb{R})$ (see [36], Theorems 24.30 and 24.31). Furthermore, the space $\mathcal{A}(\mathbb{R})$ is reflexive.

A key element of the proofs of Theorems 1 and 2 is a description of the dual space of $\mathcal{A}(\mathbb{R})$. The so-called Grothendieck-Köthe-Silva (see [30], pp. 372-378, also [1], Theorem 1.3.5) duality says that

$$
H([-n, n])_{b}^{\prime} \cong H_{0}\left(\mathbb{C}_{\infty} \backslash[-n, n]\right) .
$$

The symbol $H_{0}\left(\mathbb{C}_{\infty} \backslash[-n, n]\right)$ stands for the space of all holomorphic functions in $\mathbb{C} \backslash[-n, n]$, which vanish at infinity with the topology of uniform convergence on compact subsets. The index $b$ in $H([-n, n])_{b}^{\prime}$ denotes that the dual space is equipped with the strong topology, i.e. the topology of uniform convergence on bounded sets in $H(K)$ (we refer the reader to [36], Chapter 23, especially p. 269 for a presentation of topologies of the dual space).

Naturally the image of $\mathcal{A}(\mathbb{R})$ under the map of restriction to $K$ is dense in $H(K)$ for any $K \subset \subset \mathbb{R}$. It follows therefore from $([20], \S 26$, Satz 1.6) that algebraically

$$
\mathcal{A}(\mathbb{R})^{\prime} \cong\left(\operatorname{limproj} H\left(K_{n}\right)\right)^{\prime} \cong \operatorname{limind} H\left(K_{n}\right)^{\prime}
$$

where $\left\{K_{n}\right\}_{n \in \mathbb{N}}$ is a compact exhaustion of the real line, for instance $K_{n}=$ $[-n, n], n \in \mathbb{R}$. Isomorphism (13) holds also topologically when the dual space of the space $\mathcal{A}(\mathbb{R})$ is considered with its strong topology and limind $H\left(K_{n}\right)^{\prime}$ is equipped with the corresponding inductive topology (cf. [18], Proposition 1.7). Thus we have

$$
\mathcal{A}(\mathbb{R})_{b}^{\prime} \cong \operatorname{limind} H_{0}\left(\mathbb{C}_{\infty} \backslash[-n, n]\right)=: H_{0}(\mathbb{C} \backslash \mathbb{R}) .
$$

Since we will work with continuous linear functionals on $\mathcal{A}(\mathbb{R})$ it is important to write down the isomorphism (14) explicitly. Namely, let $\xi$ be a continuous linear functional on $\mathcal{A}(\mathbb{R})$. Then there exists an interval $[-n, n]$ and a function $f \in H_{0}\left(\mathbb{C}_{\infty} \backslash[-n, n]\right)$ such that for any $g \in \mathcal{A}(\mathbb{R})$,

$$
\xi(g)=\xi_{f}(g)=\frac{1}{2 \pi i} \int_{\gamma} f(z) G(z) d z .
$$

The function $G$ is holomorphic in some open simply connected neighborhood of $\mathbb{R}$ and restricted to $\mathbb{R}$ is equal to $g$. The $C^{\infty}$ smooth Jordan curve $\gamma$ lies in the common domain of holomorphy of $f$ and $G$, and surrounds $[-n, n]$, i.e. $[-n, n] \subset I(\gamma)$. One easily observes that the formula (15) is correct, i.e. it 
does not depend on $\gamma$ and $G$ used to compute $\xi(g)$. It also does not depend on the representative of the class of $f$ in limind $H_{0}\left(\mathbb{C}_{\infty} \backslash[-n, n]\right)$.

\subsection{Symbol Space and Toeplitz Operators}

The symbol space $\mathcal{X}(\mathbb{R})$ is defined as the inductive limit of the spaces $H(U \backslash K)$,

$$
\mathcal{X}(\mathbb{R}):=\operatorname{limind} H(U \backslash K),
$$

where $U$ runs through all open neighborhoods of $\mathbb{R}$ and $K$ through all compact subsets of $\mathbb{R}$. Definition (16) makes $\mathcal{X}(\mathbb{R})$ a locally convex space (this was discussed in detail in [9], Sect. 3). As we have already written in the Introduction, any function $\tilde{F} \in H(U \backslash K)$ determines the equivalence class $[\tilde{F}]_{\sim}$ in $\mathcal{X}(\mathbb{R})$. We call such a function a symbol function, its equivalence class is a symbol. Two such functions $\tilde{F}_{i} \in H\left(U_{i} \backslash K_{i}\right), i=1,2$ for possibly different sets $U_{i} \supset \mathbb{R}$ and $K_{i} \subset \mathbb{R}$ determine the same element of $\mathcal{X}(\mathbb{R})$ if and only if there is an open set $U \subset U_{1} \cap U_{2}$ with $\mathbb{R} \subset U$ and a compact set a compact set $K \supset K_{1} \cap K_{2}$ contained in $\mathbb{R}$ such that

$$
\left.\tilde{F}_{1}\right|_{U \backslash K}=\left.\tilde{F}_{2}\right|_{U \backslash K}
$$

One easily notices that the space $\mathcal{X}(\mathbb{R})$ is an algebra. Thus for any $F \in \mathcal{X}(\mathbb{R})$ the operator of multiplication $M_{F}: \mathcal{X}(\mathbb{R}) \rightarrow \mathcal{X}(\mathbb{R})$ is well-defined. Also, the space of all real analytic functions on $\mathbb{R}$ is a subspace of $\mathcal{X}(\mathbb{R})$.

We recall now the definition of the Cauchy transform on the space $\mathcal{X}(\mathbb{R})$ following the presentation in [28]. Let $F \in \mathcal{X}(\mathbb{R})$ and let $F$ be the equivalence class of a function $\tilde{F} \in H(U \backslash K)$, i.e. $F=[\tilde{F}]_{\sim}$, where $U$ is an open neighborhood of $\mathbb{R}$ and $K$ is a compact subset of $\mathbb{R}$. As usual we may assume that $U$ is simply connected. Let now $z \in U$ and choose a $C^{\infty}$ smooth Jordan curve in $U \backslash K$ such that both $z$ and $K$ are contained in $I(\gamma)$. We put

$$
(\mathcal{C} F)(z):=\frac{1}{2 \pi i} \int_{\gamma} \frac{\tilde{F}(\zeta)}{\zeta-z} d \zeta .
$$

Naturally, for any $z \in U$ we can find a $C^{\infty}$ smooth Jordan curve $\gamma \subset U \backslash K$ such that $z$ and $K$ are in $I(\gamma)$. By Cauchy's Theorem the value of $(\mathcal{C} F)(z)$ does not depend on $\gamma$. Hence (17) defines a function holomorphic in $U$. Its equivalence class $[\mathcal{C} F]_{\sim}$ in $\mathcal{X}(\mathbb{R})$ is by definition the Cauchy transform of $F$. We use the same symbol, that is $\mathcal{C} F$, to denote this object. One easily shows that $\mathcal{C} F \in \mathcal{X}(\mathbb{R})$ does not depend on the representative $\tilde{F}$ chosen to compute $(\mathcal{C} F)(z)$ in $(17)$. As we have already stated, $(\mathcal{C} F)(z)$ in $(17)$ is holomorphic on some neighborhood of $\mathbb{R}$. Hence its restriction to the real line belongs to $\mathcal{A}(\mathbb{R})$.

By Cauchy's integral formula, for any $F \in \mathcal{A}(\mathbb{R})$ it holds that $\mathcal{C} F=F$. That is, we have $\mathcal{C}^{2}=\mathcal{C}$ and $\mathcal{C}$ is a continuous linear projection onto $\mathcal{A}(\mathbb{R})$. The existence of a continuous projection onto $\mathcal{A}(\mathbb{R})$ readily implies that $\mathcal{A}(\mathbb{R})$ is a closed subspace of $\mathcal{X}(\mathbb{R})$. Also, $I-\mathcal{C}$ is a projection onto $H_{0}(\mathbb{C} \backslash \mathbb{R})$. Hence this space is also a closed subspace of $\mathcal{X}(\mathbb{R})$. Furthermore, it is essentially a consequence of Cauchy's integral formula that

$$
\mathcal{X}(\mathbb{R}) \cong \mathcal{A}(\mathbb{R}) \oplus H_{0}(\mathbb{C} \backslash \mathbb{R}) \cong \mathcal{A}(\mathbb{R}) \oplus \mathcal{A}(\mathbb{R})^{\prime} .
$$


We again refer the reader to ([9], Sect. 3) for the details.

For any $F \in \mathcal{X}(\mathbb{R})$ we consider the operator

$$
T_{F}:=\mathcal{C} \circ M_{F}: \mathcal{A}(\mathbb{R}) \rightarrow \mathcal{A}(\mathbb{R})
$$

and call it a Toeplitz operator. As we explained in the Introduction in (7) and (8), the matrix associated with the operator $T_{F}$ is a Toeplitz matrix. This justifies the name of this class of operators.

We now formulate explicitly the formula for the operator $T_{F}, F \in \mathcal{X}(\mathbb{R})$. Assume that $f \in \mathcal{A}(\mathbb{R})$ and let $\tilde{f} \in H(V)$ be an extension of $f$. Assume that $F$ is the equivalence class of a function $\tilde{F} \in H(U \backslash K)$, i.e. $F=[\tilde{F}]_{\sim}$. Here, $U, V$ are open neighborhoods of $\mathbb{R}$ and $K$ is a compact subset of $\mathbb{R}$. Let $W \subset U \cap V$ be a simply connected (meaning also connected) open neighborhood of $\mathbb{R}$. For $z \in W$ choose a $C^{\infty}$ smooth Jordan curve $\gamma$ contained in $W$ such that both the point $z$ and the set $K$ are contained in the interior $I(\gamma)$ of the curve $\gamma$. For any $z \in W$ such a curve exists. The function

$$
z \mapsto \frac{1}{2 \pi i} \int_{\gamma} \frac{\tilde{F}(\zeta) \cdot \tilde{f}(\zeta)}{\zeta-z} d \zeta
$$

is therefore holomorphic in $W$-by Cauchy's theorem the definition does not depend on $\gamma$. Its restriction to $\mathbb{R}$ is a real analytic function and is precisely equal to $\left(\mathcal{C} M_{F}\right) f$. We slightly abuse the notation and denote the holomorphic function defined by (18) also by $T_{F} f$. That is, $T_{F} f$ is now both an element of $\mathcal{A}(\mathbb{R})$ and its holomorphic extension to a simply connected neighborhood of $\mathbb{R}$ defined by (18).

In ([28], Sect. 3) there is provided a detailed discussion of the definition of Toeplitz operators (we emphasize however that therein (18) was used as a definition of a Toeplitz operator). In particular, it was shown that the formula is correct (see [28], Proposition 3.1). That is, it does not depend on the extension $\tilde{f} \in H(V)$ of $f \in \mathcal{A}(\mathbb{R})$ and the representative $\tilde{F} \in H(U \backslash K)$. It is also an immediate consequence of the definitions that $T_{F}: \mathcal{A}(\mathbb{R}) \rightarrow \mathcal{A}(\mathbb{R})$ is a continuous linear operator on the space of real analytic functions (see [9], Sect. 3 for the details).

We remark that sometimes to simplify the notation we will use the same symbol for a function $f \in \mathcal{A}(\mathbb{R})$ and its holomorphic extension on some open neighborhood of the line $\mathbb{R}$. Similarly, we use the same symbol to denote a class $F \in \mathcal{X}(\mathbb{R})$ and its representative in a certain space $H(U \backslash K)$.

As we have already mentioned, in the previous work [9] the authors characterized Fredholm Toeplitz operators (Theorem 2.1 above). Also, in [27] there was proved an analog of the Coburn-Simenenko Theorem for Toeplitz operators on the space of real analytic functions.

Theorem 3.1. ([27], Theorem 1.2) Assume that $T_{F}: \mathcal{A}(\mathbb{R}) \rightarrow \mathcal{A}(\mathbb{R}), F \in$ $\mathcal{X}(\mathbb{R})$ is a Toeplitz operator. Either $T_{F}$ or its adjoint $T_{F}^{\prime}$ is injective.

This result will also be important in our arguments below. 


\subsection{Wiener-Hopf Factorization}

Here we prove a result which is an analog of the Wiener-Hopf factorization. For information on this important result for Hardy spaces of Carleson curves we refer the reader to ([3], Chapter 6.12). The proof which we will give below is actually similar to the classical case ([3], Theorem 6.32). We include it for completeness.

Theorem 3.2. A Toeplitz operator $T_{F}: \mathcal{A}(\mathbb{R}) \rightarrow \mathcal{A}(\mathbb{R}), F \in \mathcal{X}(\mathbb{R})$ is a Fredholm operator if and only if $F$ admits a Wiener-Hopf factorization in $\mathcal{X}(\mathbb{R})$. That is

$$
F=F_{-} z^{k} F_{+}
$$

where $F_{+} \in \mathcal{A}(\mathbb{R}), F_{-} \in H\left(\mathbb{C}_{\infty} \backslash \mathbb{R}\right)$ do not vanish (as elements of $\mathcal{X}(\mathbb{R})$ ) and $k=-\operatorname{index} T_{F}$. In other words, it holds that $F_{+}, F_{+}^{-1} \in \mathcal{A}(\mathbb{R})$ and $F_{-}, F_{-}^{-1} \in$ $H\left(\mathbb{C}_{\infty} \backslash \mathbb{R}\right)$.

We used the symbol $H\left(\mathbb{C}_{\infty} \backslash \mathbb{R}\right)$ to denote the space

$$
\text { limind } H\left(\mathbb{C}_{\infty} \backslash K\right) \text {, }
$$

where $K$ runs through compact subsets of $\mathbb{R}$.

Proof. Assume that $T_{F}$ is a Fredholm operator with index $k$. Put $G:=F z^{k}$. Then

$$
\begin{aligned}
\operatorname{index} T_{G} & =\operatorname{index} T_{F z^{k}}=- \text { winding } F z^{k} \\
& =- \text { winding } F-k=\operatorname{index} T_{F}-k=0 .
\end{aligned}
$$

Similarly,

$$
\operatorname{index} T_{G^{-1}}=- \text { winding } F^{-1} z^{-k}=\operatorname{winding} F+k=-\operatorname{index} T_{F}+k=0 .
$$

It follows from Theorem 3.1 that both $T_{G}$ and $T_{G^{-1}}$ are invertible. Let $\mathcal{Q}:=$ $I-\mathcal{C}$ be the complementary projection to the Cauchy projection. One easily shows that the operators

$$
M_{G} \mathcal{C}+\mathcal{Q}, M_{G^{-1}} \mathcal{C}+\mathcal{Q}: \mathcal{X}(\mathbb{R}) \rightarrow \mathcal{X}(\mathbb{R})
$$

are invertible. Let $\varphi, \psi \in \mathcal{X}(\mathbb{R})$ be the solutions to the equations

$$
\left(M_{G} \mathcal{C}+\mathcal{Q}\right) \varphi=1,\left(M_{G^{-1}} \mathcal{C}+\mathcal{Q}\right) \psi=1 .
$$

We put

$$
\varphi_{+}:=\mathcal{C} \varphi, \psi_{+}:=\mathcal{C} \psi
$$

Hence

$$
G \varphi_{+}=1-\mathcal{Q} \varphi, G^{-1} \psi_{+}=1-\mathcal{Q} \psi
$$

with $\mathcal{Q} \varphi, \mathcal{Q} \psi \in H_{0}\left(\mathbb{C}_{\infty} \backslash \mathbb{R}\right)$. It follows from Liouville's theorem that $\mathcal{A}(\mathbb{R}) \cap$ $H\left(\mathbb{C}_{\infty} \backslash \mathbb{R}\right)=\{$ constant functions $\}$. Since $\varphi_{+} \psi_{+}=(1-\mathcal{Q} \varphi)(1-\mathcal{Q} \psi)$ and $(1-\mathcal{Q} \varphi)(1-\mathcal{Q} \psi)(\infty)=1$ we get $\varphi_{+} \psi_{+}=$constant $=1$.

Finally we put

$$
F_{+}=\psi_{+}, F_{-}=1-\mathcal{Q} \varphi .
$$


Then $F_{+}^{-1}=\varphi_{+} \in \mathcal{A}(\mathbb{R})$ and $F_{-}^{-1}=1-\mathcal{Q} \psi \in H\left(\mathbb{C}_{\infty} \backslash \mathbb{R}\right)$. Furthermore,

$$
\begin{aligned}
F_{-} z^{-k} F_{+} & =(1-\mathcal{Q} \varphi) z^{-k} \psi_{+}=(1-\mathcal{Q} \varphi) z^{-k} G G^{-1} \psi_{+} \\
& =(1-\mathcal{Q} \varphi) z^{-k} G(1-\mathcal{Q} \psi)=z^{-k} G=F .
\end{aligned}
$$

If $F=F_{+} z^{k} F_{-}$with $F_{+} \in \mathcal{A}(\mathbb{R})$ and $F_{-} \in H\left(\mathbb{C}_{\infty} \backslash \mathbb{R}\right)$ which do not vanish, then $F$ does not vanish and, as a result, $T_{F}$ is a Fredholm operator. Obviously, $T_{F_{+}}^{-1}=T_{F_{+}^{-1}}$ and

$$
\begin{aligned}
T_{F_{-}} T_{F_{-}^{-1}} & =\left(I-T_{\mathcal{Q} \varphi}\right)\left(I-T_{\mathcal{Q} \psi}\right)=I-T_{\mathcal{Q} \varphi}-T_{\mathcal{Q} \psi}+T_{\mathcal{Q} \varphi} T_{\mathcal{Q} \psi} \\
& =I-T_{\mathcal{Q} \varphi}-T_{\mathcal{Q} \psi}+T_{(\mathcal{Q} \varphi) \cdot(\mathcal{Q} \psi)} .
\end{aligned}
$$

This follows from the fact that $T_{F} T_{G}=T_{F G}$ if $F \in H_{0}\left(\mathbb{C}_{\infty} \backslash \mathbb{R}\right)$ or $G \in \mathcal{A}(\mathbb{R})$. Hence

$$
T_{F_{-}} T_{F_{-}}^{-1}=T_{(1-\mathcal{Q} \varphi)(1-\mathcal{Q} \psi)}=I .
$$

We infer that also $T_{F_{-}}$is invertible and, as a result, is a Fredholm operator of index 0 .

\section{Proofs of the Main Results}

\subsection{Left Invertibility of Toeplitz Operators}

We prove here our first main result. In order to explain and motivate our methods we shall deal first with the multiplication operator on $\mathcal{A}(\mathbb{R})$ by a function in $\mathcal{A}(\mathbb{R})$, which is naturally an example of a Toeplitz operator.

Theorem 4.1. Let $\left(a_{n}\right) \subset \mathbb{R}$ be a sequence converging to $\infty$. The space

$$
Y:=\left\{f \in \mathcal{A}(\mathbb{R}): f\left(a_{n}\right)=0\right\}
$$

is not complemented in $\mathcal{A}(\mathbb{R})$.

As we remarked above, this theorem is given to motivate the general method. This is why we only sketch the proof.

Proof. For each $N \in \mathbb{N}$ define the space

$$
X_{N}:=\left\{f \in \mathcal{A}(\mathbb{R}): f\left(a_{N}\right)=f\left(a_{N+1}\right)=\cdots=0\right\} .
$$

Let also $X:=\bigcup X_{N} \subset \mathcal{A}(\mathbb{R})$. Choose functions $f_{n} \in \mathcal{A}(\mathbb{R})$ such that $f_{n}\left(a_{m}\right)=\delta_{n m}$. For $f \in X$ the sum

$$
\sum f\left(a_{n}\right) f_{n}
$$

is well-defined, since it is a finite sum. Also for any $f \in X$ the function

$$
f-\sum f\left(a_{n}\right) f_{n}
$$

belongs to $Y$. Assume that $Y$ is complemented and let $P: \mathcal{A}(\mathbb{R}) \rightarrow Y$ be a continuous projection onto $Y$. Since for any $f \in X$ the function $f-\sum f\left(a_{n}\right) f_{n}$ is in $Y$, we have

$$
P\left(f-\sum f\left(a_{n}\right) f_{n}\right)=f-\sum f\left(a_{n}\right) f_{n}
$$


and

$$
(I-P) f=f-P f=\sum f\left(a_{n}\right)\left(f_{n}-P f_{n}\right) .
$$

Recall that the sum is finite. This shows that if $Y$ is complemented, then there exist functions $\varphi_{n} \in \mathcal{A}(\mathbb{R})$ such that for any $f \in X$,

$$
(I-P) f=\sum f\left(a_{n}\right) \varphi_{n}
$$

Note that no function $\varphi_{n}$ vanishes identically. Indeed, if $\varphi_{n} \equiv 0$, then $f_{n}=$ $P f_{n}$, which implies that $f_{n} \in Y$. This is impossible, since $f_{n}\left(a_{n}\right)=1$. There exists therefore a point $\tilde{z} \in \mathbb{R}$ such that $\varphi_{n}(\tilde{z}) \neq 0$ for all $n \in \mathbb{N}$. Choose a function $f \in H(\mathbb{C})$ such that

$$
f\left(a_{n}\right)=\frac{1}{\varphi_{n}(\tilde{z})}, n \in \mathbb{N} .
$$

Such a function exists by the Weierstrass Theorem. Let

$$
F_{N}(z):=\prod_{n=N+1}^{\infty} E_{p_{n}}\left(\frac{z}{a_{n}}\right)
$$

with appropriately chosen numbers $p_{n}$. Consider the function $f F_{N}$. The sequence of functions $g_{N}=f F_{N}$ converges in $H(\mathbb{C})$ to $f$. Hence, their restrictions to the real line converge to $\left.f\right|_{\mathbb{R}}$ in $\mathcal{A}(\mathbb{R})$. Since $P$ is continuous, $P\left(\left.g_{N}\right|_{\mathbb{R}}\right) \rightarrow P\left(\left.f\right|_{\mathbb{R}}\right)$ and $(I-P)\left(\left.g_{N}\right|_{\mathbb{R}}\right) \rightarrow(I-P)\left(\left.f\right|_{\mathbb{R}}\right)$. On the other hand,

$$
(I-P)\left(\left.g_{N}\right|_{\mathbb{R}}\right)=\sum g_{N}\left(a_{n}\right) \varphi_{n}
$$

since $\left.g_{N}\right|_{\mathbb{R}} \in X$. We have

$$
\begin{aligned}
(I-P)\left(g_{N} \mid \mathbb{R}_{R}\right)(\tilde{z}) & =\sum f\left(a_{n}\right) F_{N}\left(a_{n}\right) \varphi_{n}(\tilde{z})=\sum \frac{1}{\varphi_{n}(\tilde{z})} F_{N}\left(a_{n}\right) \varphi_{n}(\tilde{z}) \\
& =\sum_{n=1}^{\infty} F_{N}\left(a_{n}\right)=F_{N}\left(a_{1}\right)+\cdots+F_{N}\left(a_{N}\right),
\end{aligned}
$$

which diverges, since $F_{N}$ converges to 1 uniformly on compact subsets of the plane. This is a contradiction, since the evaluation at $\tilde{z}$ is a continuous functional on $\mathcal{A}(\mathbb{R})$. The formal argument requires a precise choice of the numbers $p_{n}$. We shall refrain from writing down the details here. This will be done in the proof of Theorem 3 .

Our aim now is to generalize the arguments from the proof of Theorem 4.1 for Toeplitz operators. We consider the operator $T_{F}$ with $F \in \mathcal{X}(\mathbb{R})$, where $F$ is the equivalence class of $\tilde{F} \in H(U \backslash K)$, with $U$ an open neighborhood of $\mathbb{R}$ and $K$ a compact subset of $\mathbb{R}$. We assume that $\tilde{F}$ vanishes only on a sequence $z_{n} \in \mathbb{R}$ which accumulates only at $\pm \infty$.

According to Theorem 2.2 the operator $T_{F}$ is injective and has closed range. We prove that the range of $T_{F}$ is not a complemented subspace of $\mathcal{A}(\mathbb{R})$. The first step is a factorization of the symbol function $\tilde{F}$. Let $m_{n}$ be the multiplicity of $z_{n}, n \in \mathbb{N}$. We assume that

$$
0<\left|z_{1}\right| \leq\left|z_{2}\right| \leq \cdots
$$


Note that we can always assume that $0 \in K$ and $z_{1} \neq 0$. Using Weierstrass theory we write

$$
\tilde{F}(z)=\prod_{n=1}^{\infty} E_{p_{n}}^{m_{n}}\left(\frac{z}{z_{n}}\right) \cdot F_{0}(z),
$$

where the sequence $\left(p_{n}\right)$ is now only assumed to be chosen in such a way that for any $r>0$

$$
\sum_{n=1}^{\infty} m_{n}\left(\frac{r}{\left|z_{n}\right|}\right)^{p_{n}+1}<\infty .
$$

In order to complete the proof of Theorem 3, a more precise choice of the sequence $\left(p_{n}\right)$ will be needed. This will be done at the end of the proof. Observe that the function $F_{0} \in H(U \backslash K)$ does not vanish in $U \backslash K$.

Consider the operator $T_{F_{0}}$. To simplify the notation we write $T_{F_{0}}$ for the Toeplitz operator corresponding to the symbol $\left[F_{0}\right]_{\sim} \in \mathcal{X}(\mathbb{R})$. We apply this convention also in the next results.

It follows from Theorem 2.1 that the operator $T_{F_{0}}$ is a Fredholm operator and

$$
\operatorname{index} T_{F_{0}}=\operatorname{dim} \operatorname{ker} T_{F_{0}}-\operatorname{dim} \operatorname{coker} T_{F_{0}}=-\operatorname{winding} F_{0} .
$$

Our first goal is to construct a sequence of continuous linear functionals $\xi_{n} \in \mathcal{A}(\mathbb{R})^{\prime}, n \in \mathbb{N}$ such that

$$
R\left(T_{F}\right)=\bigcap_{n=1}^{\infty} \operatorname{ker} \xi_{n} .
$$

Any $\xi$ is $\xi_{\varphi}($ see $(15))$ for a function $\varphi \in H_{0}\left(\mathbb{C}_{\infty} \backslash L\right), L$ a compact subset of $\mathbb{R}$. In order to simplify the notation we shall identify the functional $\xi_{\varphi}$ with the function $\varphi$. With this convention (20) takes the form

$$
R\left(T_{F}\right)=\bigcap_{n=1}^{\infty} \operatorname{ker} \varphi_{n},
$$

for some sequence $\varphi_{n} \in H_{0}\left(\mathbb{C}_{\infty} \backslash L_{n}\right), L_{n} \subset \subset \mathbb{R}$. The following fact is a key to many results proved below. Roughly speaking continuous linear functionals cannot distinguish between a multiplication operator and a general Toeplitz operator.

Lemma 4.2. Assume that $F \in \mathcal{X}(\mathbb{R}), F=[\tilde{F}]_{\sim}$ for $\tilde{F} \in H(U \backslash K), f \in \mathcal{A}(\mathbb{R})$ with $f=\left.\tilde{f}\right|_{\mathbb{R}}$ for $\tilde{f} \in H(V)$ and $\varphi \in H_{0}\left(\mathbb{C}_{\infty} \backslash L\right)$. Then

$$
\left\langle T_{F} f, \xi_{\varphi}\right\rangle=\left\langle T_{F} f, \varphi\right\rangle=\int_{\gamma} \tilde{F} \tilde{f} \varphi d z,
$$

where $\gamma$ is a $C^{\infty}$ smooth Jordan curve in $W \backslash(K \cup L)$ such that $K \cup L \subset I(\gamma)$. The symbol $W$ stands for a simply connected neighborhood of $\mathbb{R}$ contained in $U \cap V$. 
Proof. The proof is an immediate consequence of Fubini's theorem. Let $W \subset$ $U \cap V$ be a simply connected neighborhood of $\mathbb{R}$. The function $T_{F} f$ defined as in (18) is holomorphic in $W$-recall our convention concerning denoting Toeplitz operators. Let $\delta$ be a $C^{\infty}$ smooth Jordan curve contained in $W$ and such that $L \subset I(\delta)$. Then

$$
\left\langle T_{F} f, \xi_{\varphi}\right\rangle=\int_{\delta}\left(T_{F} f\right)(z) \varphi(z) d z
$$

Choose a $C^{\infty}$ smooth Jordan curve $\gamma$ in $W$ such that $K \subset I(\gamma)$ and also $\delta \subset I(\gamma)$. We have

$$
\begin{aligned}
\left\langle T_{F} f, \varphi\right\rangle & =\int_{\delta}\left(\int_{\gamma} \frac{\tilde{F}(\zeta) \tilde{f}(\zeta)}{\zeta-z} d \zeta\right) \varphi(z) d z \\
& =\int_{\gamma} \tilde{F}(\zeta) \tilde{f}(\zeta) \int_{\delta} \frac{\varphi(z)}{\zeta-z} d z d \zeta=\int_{\gamma} \tilde{F}(\zeta) \tilde{f}(\zeta) \varphi(\zeta) d \zeta
\end{aligned}
$$

by Cauchy's integral formula, since $\varphi$ vanishes at $\infty$.

The next proposition is a key technical ingredient in the proof of Theorem 3. Let us recall that $F_{0}$ is holomorphic in $U \backslash K$. We may assume without loss of generality that $U$ is simply connected.

Proposition 4.3. If index $T_{F_{0}}=-1$, then there exists a function $\varphi \in H_{0}\left(\mathbb{C}_{\infty} \backslash L\right), L$ a compact subset of $\mathbb{R}$, such that

$$
R\left(T_{F}\right)=\operatorname{ker} \varphi \cap \bigcap_{n=1}^{\infty} \bigcap_{k=1}^{m_{n}} \operatorname{ker} \frac{\varphi}{\left(z-z_{n}\right)^{k}}
$$

If index $T_{F_{0}}=k \geq 0$, then there exists a function $\varphi \in H_{0}\left(\mathbb{C}_{\infty} \backslash L\right), L a$ compact subset of $\mathbb{R}$, and natural numbers $l, \mu_{l}, \mu_{l} \leq m_{l}$, such that

$R\left(T_{F}\right)=\operatorname{ker} \varphi \cap \operatorname{ker} \frac{\varphi}{z-z_{l}} \cap \cdots \cap \operatorname{ker} \frac{\varphi}{\left(z-z_{l}\right)^{m_{l}-\mu_{l}}} \cap \bigcap_{i=l+1}^{\infty} \bigcap_{j=1}^{m_{i}} \operatorname{ker} \frac{\varphi}{\left(z-z_{i}\right)^{j}}$.

If index $T_{F_{0}}=k<-1$, then there exists a function $\varphi \in H_{0}\left(\mathbb{C}_{\infty} \backslash L\right), L a$ compact subset of $\mathbb{R}$, and natural numbers $l, \mu_{l}$ such that

$$
\begin{aligned}
& R\left(T_{F}\right) \\
& =\operatorname{ker} \varphi \cap \bigcap_{j=1}^{m_{l}+\mu_{l}} \operatorname{ker} \frac{\varphi}{\left(z-z_{l}\right)^{j}} \cap \bigcap_{i=1}^{l-1} \bigcap_{j=1}^{2 m_{i}} \operatorname{ker} \frac{\varphi}{\left(z-z_{i}\right)^{j}} \cap \bigcap_{i=l+1}^{\infty} \bigcap_{j=1}^{m_{i}} \operatorname{ker} \frac{\varphi}{\left(z-z_{i}\right)^{j}} .
\end{aligned}
$$

Also, if index $T_{F_{0}} \leq-1$, then there exists a function $a \in H(U)$ such that

$$
\varphi(z)=\frac{a(z)}{F_{0}(z)}
$$

for $z \in U \backslash(K \cup L)$.

If index $T_{F_{0}} \geq 0$, then there exists a function $a \in H(U)$ such that

$$
\varphi(z)=\frac{1}{\prod_{n=1}^{l-1} E_{p_{n}}^{m_{n}}\left(\frac{z}{z_{n}}\right) \cdot E_{p_{l}}^{\mu_{l}}\left(\frac{z}{z_{n}}\right)} \frac{a(z)}{F_{0}(z)}
$$

for $z \in U \backslash(K \cup L)$ and $z \neq z_{1}, \ldots, z_{l}$. 
To prepare the proof of Proposition 4.3 we need some lemmas. The next lemma in accordance with Lemma 4.2 explains the reason why (21) and also (22) hold true.

Lemma 4.4. Assume that $G \in H(U \backslash K), U$ is an open simply connected neighborhood of $\mathbb{R}, K$ is a compact subset of $\mathbb{R}$. If for every polynomial $p$

$$
\int_{\gamma} G p d z=0
$$

where $\gamma$ is a $C^{\infty}$ smooth Jordan curve in $U \backslash K$ with $K \subset I(\gamma)$, then $G \in$ $H(U)$.

Proof. By Cauchy's integral formula $G=G_{+}+G_{-}$, where $G_{+} \in H(U)$ and $G_{-} \in H_{0}\left(\mathbb{C}_{\infty} \backslash K\right)$. We have

$$
0=\int_{\gamma} G p d z=\int_{\gamma}\left(G_{+}+G_{-}\right) p d z=\int_{\gamma} G_{-} p d z=\int_{|z|=R}\left(\sum_{n=1}^{\infty} \frac{G_{-n}}{z^{n}}\right) p(z) d z,
$$

for sufficiently large $R>0$, by Cauchy's theorem. This implies that $G_{-n}=0$ for $n \in \mathbb{N}$. Hence, $G=G_{+}$.

Our method to prove Proposition 4.3 is to reduce the proof to the finite dimensional case of Fredholm operators. We formulate now three auxiliary Lemmas.

Lemma 4.5. Assume that $F_{0} \in H(U \backslash K)$ does not vanish, $U$ is an open simply connected neighborhood of $\mathbb{R}, K$ is a compact subset of $\mathbb{R}$. Furthermore, assume that index $T_{F_{0}}=-1$. Then there exists a function $\varphi \in H_{0}\left(\mathbb{C}_{\infty} \backslash L\right)$ such that

$$
R\left(T_{F_{0}}\right)=\operatorname{ker} \varphi
$$

Proof. Since index $T_{F_{0}}<0$, it must hold that dim coker $T_{F_{0}}>0$. The operator $T_{F_{0}}$ is a Fredholm operator (see Theorem 2.1). Hence its range is a closed subspace of $\mathcal{A}(\mathbb{R})$ ([9], Proposition 5.1). There exists therefore a non-zero functional which vanishes on $R\left(T_{F_{0}}\right)$. Such a functional belongs to $\operatorname{ker} T_{F_{0}}^{\prime}$. In view of the Coburn-Simonenko theorem (Theorem 3.1), it must hold that $\operatorname{ker} T_{F_{0}}=\{0\}$. We infer that dimcoker $T_{F_{0}}=1$. Let a functional $\xi$ span $\left(\mathcal{A}(\mathbb{R}) / R\left(T_{F_{0}}\right)\right)^{\prime}$. Define $\tilde{\xi}:=\xi \circ \pi$, where $\pi: \mathcal{A}(\mathbb{R}) \rightarrow \mathcal{A}(\mathbb{R}) / R\left(T_{F_{0}}\right)$ is the quotient map. Obviously $R\left(T_{F_{0}}\right) \subset \operatorname{ker} \xi$ and both spaces are of codimension one. It must be that $R\left(T_{F_{0}}\right)=\operatorname{ker} \xi$.

Lemma 4.6. Assume that $F_{0} \in H(U \backslash K)$ does not vanish, $U$ is an open simply connected neighborhood of $\mathbb{R}, K$ is a compact subset of $\mathbb{R}$. Furthermore, assume that

$$
\operatorname{index} T_{F_{0}}=- \text { winding } F_{0}=-1 \text {. }
$$

Let $\varphi \in H_{0}\left(\mathbb{C}_{\infty} \backslash L\right)$ be as in the previous lemma, i.e.

$$
R\left(T_{F_{0}}\right)=\operatorname{ker} \varphi \text {. }
$$


For $N \in \mathbb{N}$ consider the symbol function

$$
G(z):=\prod_{n=1}^{N-1} E_{p_{n}}^{m_{n}}\left(\frac{z}{z_{n}}\right) \cdot E_{p_{N}}^{l}\left(\frac{z}{z_{n}}\right) \cdot F_{0}(z):=\mathcal{E}(z) \cdot F_{0}(z),
$$

with $l \leq m_{N}$. Then

$R\left(T_{G}\right)=\operatorname{ker} \varphi \cap \bigcap_{n=1}^{N-1} \bigcap_{k=1}^{m_{n}} \operatorname{ker} \frac{\varphi}{\left(z-z_{n}\right)^{k}} \cap \operatorname{ker} \frac{\varphi}{\left(z-z_{N}\right)} \cap \cdots \cap \operatorname{ker} \frac{\varphi}{\left(z-z_{N}\right)^{l}}$.

In other words, if $\xi_{\psi}$ vanishes on $R\left(T_{G}\right)$, then

$$
\begin{aligned}
\psi \in & \operatorname{span}\left\{\varphi, \frac{\varphi}{\left(z-z_{1}\right)}, \ldots, \frac{\varphi}{\left(z-z_{1}\right)^{m_{1}}}, \ldots, \frac{\varphi}{\left(z-z_{N-1}\right)}, \ldots, \frac{\varphi}{\left(z-z_{N-1}\right)^{m_{N-1}}},\right. \\
& \left.\frac{\varphi}{\left(z-z_{N}\right)}, \ldots, \frac{\varphi}{\left(z-z_{N}\right)^{l}}\right\} .
\end{aligned}
$$

Proof. There is a finite number of zeros of the symbol function $G$ in $U \backslash K$, all of them are real. There is therefore a compact set $\tilde{K} \subset \mathbb{R}$ such that $G$ does not vanish in $U \backslash \tilde{K}$. It follows therefore from Theorem 2.1 that $T_{G}$ is a Fredholm operator. Furthermore, by the argument principle we have

$$
\begin{aligned}
\operatorname{index} T_{G} & =- \text { winding } G=-\left(m_{1}+\cdots+m_{N-1}+l+\text { winding } F_{0}\right) \\
& =-\left(m_{1}+\cdots+m_{N-1}+l\right)+\operatorname{index} T_{F_{0}} \\
& =-\left(m_{1}+\cdots+m_{N-1}+l\right)-1 .
\end{aligned}
$$

As in the proof of Lemma 4.5 it follows from the Coburn-Simonenko theorem that $\operatorname{dim} \operatorname{ker} T_{G}=0$ and

$$
\operatorname{dim} \text { coker } T_{G}=\sum_{n=1}^{N-1} m_{n}+l+1 .
$$

Consider the family of functions

$$
\begin{aligned}
\mathcal{G}:= & \left\{\varphi, \frac{\varphi}{\left(z-z_{1}\right)}, \ldots, \frac{\varphi}{\left(z-z_{1}\right)^{m_{1}}}, \ldots, \frac{\varphi}{\left(z-z_{N-1}\right)}, \ldots, \frac{\varphi}{\left(z-z_{N-1}\right)^{m_{N-1}}},\right. \\
& \left.\frac{\varphi}{\left(z-z_{N}\right)}, \ldots, \frac{\varphi}{\left(z-z_{N}\right)^{l}}\right\} .
\end{aligned}
$$

Each of these functions defines a continuous linear functional on $\mathcal{A}(\mathbb{R})$, since they belong to $H_{0}\left(\mathbb{C}_{\infty} \backslash\left(L \cup\left\{z_{1}, \ldots, z_{N}\right\}\right)\right)$ and $z_{1}, \ldots, z_{N}$ are real. The number of the functions is equal to $\operatorname{dim} \operatorname{coker} T_{G}$. We claim that the continuous linear functionals defined by functions in $\mathcal{G}$ all vanish on $R\left(T_{G}\right)$, i.e.

$$
R\left(T_{G}\right) \subset \bigcap_{\psi \in \mathcal{G}} \operatorname{ker} \psi
$$

Let us apply Lemma 4.2. Let $\psi \in \mathcal{G}$ and $f \in \mathcal{A}(\mathbb{R})$-we again simplify the notation and denote an extension of $f$ to some open neighborhood of $\mathbb{R}$ by the same symbol $f$. For some $n \in\{1, \ldots, N-1\}$ and $j \leq m_{n}$ or $n=N$ and $j \leq l$ we have 


$$
\begin{aligned}
\left\langle T_{G} f, \xi_{\psi}\right\rangle & =\int_{\gamma} G f \psi d z=\int_{\gamma} F_{0}(z) \cdot \frac{\mathcal{E}(z)}{\left(z-z_{n}\right)^{j}} \cdot f(z) \varphi(z) d z \\
& =\left\langle T_{F_{0}}(g), \xi_{\varphi}\right\rangle
\end{aligned}
$$

for the function $g=\frac{\mathcal{E}}{\left(z-z_{i}\right)^{j}} \cdot f \in \mathcal{A}(\mathbb{R})$. The curve $\gamma$ is a $C^{\infty}$ smooth Jordan curve contained in the intersection of the domains of $F_{0}$ and $f$, which may be assumed to be simply connected, and such that the sets $K, L$ and the points $z_{1}, \ldots, z_{N}$ are contained in $I(\gamma)$. Hence,

$$
\left\langle T_{G} f, \xi_{\psi}\right\rangle=0
$$

for every $f \in \mathcal{A}(\mathbb{R})$ and every $\psi \in \mathcal{G}$, since $\xi_{\varphi}$ vanishes on the range of $T_{F_{0}}$. In order to complete the proof of the lemma it suffices to show that the functions in $\mathcal{G}$ are linearly independent. This is however elementary.

We now consider the case

$$
\operatorname{index} T_{F_{0}}=k \geq 0 \text {. }
$$

Find a number $l \in \mathbb{N}$ and $\mu_{l}$ with $0 \leq \mu_{l} \leq m_{l}$ such that

$$
m_{0}+\cdots+m_{l-1}+\mu_{l}=k+1,
$$

with the convention that $m_{0}=0$. Consider the symbol function

$$
G(z):=\prod_{n=1}^{l-1} E_{p_{n}}^{m_{n}}\left(\frac{z}{z_{n}}\right) E_{p_{l}}^{\mu_{l}}\left(\frac{z}{z_{l}}\right) F_{0}(z) .
$$

It follows from Theorem 2.1 that

$\operatorname{index} T_{G}=-$ winding $G=-\left(m_{0}+\cdots+m_{l-1}+\mu_{l}\right)+\operatorname{index} T_{F_{0}}=-1$.

In view of Lemma 4.5 there exists $\varphi \in H_{0}\left(\mathbb{C}_{\infty} \backslash L\right)$ with a compact $L \subset \mathbb{R}$ such that

$$
R\left(T_{G}\right)=\operatorname{ker} \xi_{\varphi}
$$

Lemma 4.7. With the above notation consider the symbol function

$$
H(z):=\prod_{n=1}^{l-1} E_{p_{n}}^{m_{n}}\left(\frac{z}{z_{n}}\right) E_{p_{l}}^{\mu_{l}+\nu_{l}}\left(\frac{z}{z_{l}}\right) \cdot F_{0}(z),
$$

where $0 \leq \nu_{l} \leq m_{l}-\mu_{l}$. Then

$$
R\left(T_{H}\right)=\operatorname{ker} \varphi \cap \operatorname{ker} \frac{\varphi}{\left(z-z_{l}\right)} \cap \cdots \cap \operatorname{ker} \frac{\varphi}{\left(z-z_{l}\right)^{\nu_{l}}} .
$$

In other words, if $\xi_{\psi}$ vanishes on $R\left(T_{H}\right)$, then

$$
\psi \in \operatorname{Span}\left\{\varphi, \frac{\varphi}{\left(z-z_{l}\right)}, \ldots, \frac{\varphi}{\left(z-z_{l}\right)^{\nu_{l}}}\right\} .
$$

Similarly, consider the symbol function

$$
H(z):=\prod_{n=1}^{N-1} E_{p_{n}}^{m_{n}}\left(\frac{z}{z_{n}}\right) \cdot E_{p_{N}}^{\nu_{N}}\left(\frac{z}{z_{N}}\right) \cdot F_{0}(z),
$$


where $N>l$ and $0 \leq \nu_{N} \leq m_{N}$. Then

$$
\begin{aligned}
R\left(T_{H}\right)= & \operatorname{ker} \varphi \cap \operatorname{ker} \frac{\varphi}{\left(z-z_{l}\right)} \cap \cdots \cap \operatorname{ker} \frac{\varphi}{\left(z-z_{l}\right)^{m_{l}-\mu_{l}}} \cap \\
& \cap \bigcap_{n=l+1}^{N-1} \bigcap_{k=1}^{m_{n}} \operatorname{ker} \frac{\varphi}{\left(z-z_{n}\right)^{k}} \cap \operatorname{ker} \frac{\varphi}{\left(z-z_{N}\right)} \cap \cdots \cap \operatorname{ker} \frac{\varphi}{\left(z-z_{N}\right)^{\nu_{N}}} .
\end{aligned}
$$

In other words, if $\xi_{\psi}$ vanishes on $R\left(T_{H}\right)$, then

$$
\begin{aligned}
\psi \in & \operatorname{Span}\left\{\varphi, \frac{\varphi}{\left(z-z_{l}\right)}, \ldots, \frac{\varphi}{\left(z-z_{l}\right)^{m_{l}-\mu_{l}}}, \frac{\varphi}{\left(z-z_{l+1}\right)}, \ldots,\right. \\
& \frac{\varphi}{\left(z-z_{l+1}\right)^{m_{l+1}}}, \ldots, \frac{\varphi}{\left(z-z_{N-1}\right)}, \ldots, \frac{\varphi}{\left(z-z_{N-1}\right)^{m_{N-1}}}, \\
& \left.\frac{\varphi}{\left(z-z_{N}\right)}, \ldots, \frac{\varphi}{\left(z-z_{N}\right)^{\nu_{N}}}\right\} .
\end{aligned}
$$

Proof. In order to prove the lemma, it suffices to apply Lemma 4.6 with $G$ instead of $F_{0}$. Indeed, there is a finite number of real zeros of $G$ in $U \backslash K$. Hence, there is a compact subset $\tilde{K} \subset \mathbb{R}$ such that $G$ does not vanish in $U \backslash \tilde{K}$. Also, index $T_{G}=-1$, we may therefore invoke Lemma 4.6. For instance when $H$ is defined by (26) we have

$$
H(z)=E_{p_{l}}^{\nu_{l}}\left(\frac{z}{z_{l}}\right) \cdot G(z)
$$

Now we investigate the case index $T_{F_{0}}=k<-1$. Choose $l \in \mathbb{N}$ and $0 \leq \mu_{l} \leq m_{l}$ such that

$$
m_{0}+m_{1}+\cdots+m_{l-1}+\mu_{l}=|k|-1 .
$$

Consider the symbol function

$$
G(z):=\frac{F_{0}(z)}{\prod_{n=1}^{l-1} E_{p_{n}}^{m_{n}}\left(\frac{z}{z_{n}}\right) \cdot E_{p_{l}}^{\mu_{l}}\left(\frac{z}{z_{l}}\right)} .
$$

Since the denominator vanishes only on a finite number of real zeros, $G$ is a symbol function, i.e. it is holomorphic in some set of the form $U \backslash \tilde{K}$. In this set the function $G$ does not vanish, since $F_{0}$ does not vanish. It follows therefore from Theorem 2.1 that $T_{G}$ is a Fredholm operator and

$$
\text { index } T_{G}=- \text { winding } G=|k|-1+\operatorname{index} T_{F_{0}}=-1 \text {. }
$$

There exists therefore $\varphi \in H_{0}\left(\mathbb{C}_{\infty} \backslash L\right)$ such that

$$
R\left(T_{G}\right)=\operatorname{ker} \xi_{\varphi} .
$$

Lemma 4.8. With the notation introduced above consider the symbol

$$
H(z):=\frac{F_{0}(z)}{\prod_{n=1}^{j-1} E_{p_{n}}^{m_{n}}\left(\frac{z}{z_{n}}\right) \cdot E_{p_{j}}^{\nu_{l}}\left(\frac{z}{z_{j}}\right)},
$$


where $1 \leq j<l, 0 \leq \nu_{j} \leq m_{j}$ or if $j=l, \nu_{l} \leq \mu_{l}$. Then the range of $T_{H}$ is the intersection of the kernels of the functionals which correspond to the functions in the family

$$
\mathcal{H}:=\left\{\varphi, \frac{\varphi}{\left(z-z_{l}\right)}, \ldots, \frac{\varphi}{\left(z-z_{l}\right)^{\mu_{l}-\nu_{l}}}\right\}
$$

if $j=l$ in (28) and to the functions in the family

$$
\begin{aligned}
\mathcal{H}:= & \left\{\varphi, \frac{\varphi}{\left(z-z_{l}\right)^{\mu_{l}}}, \ldots, \frac{\varphi}{\left(z-z_{l}\right)}, \frac{\varphi}{\left(z-z_{l-1}\right)^{m_{l-1}}}, \ldots, \frac{\varphi}{\left(z-z_{l-1}\right)}, \ldots,\right. \\
& \left.\frac{\varphi}{\left(z-z_{j}\right)^{m_{j}-\nu_{j}}}, \ldots, \frac{\varphi}{\left(z-z_{j}\right)}\right\}
\end{aligned}
$$

if $j<l$.

Consider the symbol

$$
H:=\prod_{n=1}^{j-1} E_{p_{n}}^{m_{n}}\left(\frac{z}{z_{n}}\right) \cdot E_{p_{j}}^{\nu_{j}}\left(\frac{z}{z_{j}}\right) \cdot F_{0}(z)
$$

for some $1 \leq j \leq l$. The range of the operator $T_{H}$ is the intersection of the kernels of the functionals which corresponds to the functions in the family

$$
\begin{aligned}
\mathcal{H}:= & \left\{\varphi, \frac{\varphi}{\left(z-z_{l}\right)^{\mu_{l}}}, \ldots, \frac{\varphi}{\left(z-z_{l}\right)}, \frac{\varphi}{\left(z-z_{l-1}\right)^{m_{l-1}}}, \ldots, \frac{\varphi}{\left(z-z_{l-1}\right)}, \ldots,\right. \\
& \frac{\varphi}{\left(z-z_{1}\right)^{m_{1}}}, \ldots, \frac{\varphi}{\left(z-z_{1}\right)}, \frac{\varphi}{\left(z-z_{1}\right)^{m_{1}+1}}, \ldots, \frac{\varphi}{\left(z-z_{1}\right)^{2 m_{1}}}, \ldots, \\
& \frac{\varphi}{\left(z-z_{j-1}\right)^{m_{j-1}+1}}, \ldots, \frac{\varphi}{\left(z-z_{j-1}\right)^{2 m_{j-1}}}, \\
& \left.\frac{\varphi}{\left(z-z_{j}\right)^{m_{j}+1}}, \ldots, \frac{\varphi}{\left(z-z_{j}\right)^{m_{j}+\nu_{j}}}\right\} .
\end{aligned}
$$

Consider the same symbol as in (29) for $j>l$. Then the range of the operator $T_{H}$ is the intersection of the kernels of the functionals which correspond to the functions in the family

$$
\begin{aligned}
\mathcal{H}:= & \left\{\varphi, \frac{\varphi}{\left(z-z_{l}\right)^{\mu_{l}}}, \ldots, \frac{\varphi}{\left(z-z_{l}\right)}, \frac{\varphi}{\left(z-z_{l-1}\right)^{m_{l-1}}}, \ldots, \frac{\varphi}{\left(z-z_{l-1}\right)}, \ldots,\right. \\
& \frac{\varphi}{\left(z-z_{1}\right)^{m_{1}}}, \ldots, \frac{\varphi}{\left(z-z_{1}\right)}, \frac{\varphi}{\left(z-z_{1}\right)^{m_{1}+1}}, \ldots, \frac{\varphi}{\left(z-z_{1}\right)^{2 m_{1}}}, \ldots, \\
& \frac{\varphi}{\left(z-z_{l-1}\right)^{m_{l-1}+1}}, \ldots, \frac{\varphi}{\left(z-z_{l-1}\right)^{2 m_{l-1}}}, \frac{\varphi}{\left(z-z_{l}\right)^{\mu_{l}+1}}, \ldots, \frac{\varphi}{\left(z-z_{l}\right)^{m_{l}+\mu_{l}}}, \\
& \frac{\varphi}{\left(z-z_{l+1}\right)}, \ldots, \frac{\varphi}{\left(z-z_{l+1}\right)^{m_{l+1}}}, \ldots, \frac{\varphi}{\left(z-z_{j-1}\right)}, \ldots, \frac{\varphi}{\left(z-z_{j-1}\right)^{m_{j-1}}}, \\
& \left.\frac{\varphi}{\left(z-z_{j}\right)}, \ldots, \frac{\varphi}{\left(z-z_{j}\right)^{\nu_{j}}}\right\} .
\end{aligned}
$$

Proof. Again in order to prove the lemma, it suffices to apply Lemma 4.6 with $G$ instead of $F_{0}$.

We are now ready to prove Proposition 4.3. 
Proof of Proposition 4.3. Assume that index $T_{F_{0}}=-1$. It follows from Lemma 4.5 that there exists $\varphi \in H_{0}\left(\mathbb{C}_{\infty} \backslash L\right)$ such that

$$
R\left(T_{F_{0}}\right)=\operatorname{ker} \varphi \text {. }
$$

We show that

$$
R\left(T_{F}\right)=\operatorname{ker} \varphi \cap \operatorname{ker} \frac{\varphi}{\left(z-z_{1}\right)} \cap \cdots \cap \operatorname{ker} \frac{\varphi}{\left(z-z_{1}\right)^{m_{1}}} \cap \operatorname{ker} \frac{\varphi}{\left(z-z_{2}\right)} \cap \cdots .
$$

The same argument as in Lemma 4.6 shows the inclusion $\subset$. Indeed, write $F=\mathcal{E} \cdot F_{0}$, then

$$
\left\langle T_{F} f, \xi_{\psi}\right\rangle=\int_{\gamma} F_{0} \frac{\mathcal{E}}{\left(z-z_{i}\right)^{j}} f \varphi d z=\left\langle T_{F_{0}} g, \varphi\right\rangle=0,
$$

with $g=\frac{\mathcal{E}}{\left(z-z_{i}\right)^{j}} f \in \mathcal{A}(\mathbb{R})$, since $\xi_{\varphi}$ vanishes on $R\left(T_{F_{0}}\right)$. We use as before the same symbol $f$ for an extension of $f \in \mathcal{A}(\mathbb{R})$ to some holomorphic function in $H(V), V$ an open neighborhood of $\mathbb{R}$. As usual $\gamma$ is a $C^{\infty}$ smooth Jordan curve in $W \backslash(K \cup L)$ with $K \cup L \subset I(\gamma)$. The symbol $W$ denotes, as in Lemma 4.2, a simply connected neighborhood of $\mathbb{R}$ contained in the intersections of the open sets $U$ and $V$. We use this convention below as well and also we write $F$ to denote not only the symbol but also the symbol function, which represents $F$.

Assume that $\xi=\xi_{\psi}$ vanishes on the range of $T_{F}$, i.e. for any $f \in \mathcal{A}(\mathbb{R})$

$$
\left\langle T_{F} f, \xi_{\psi}\right\rangle=0 \text {. }
$$

In other words,

$$
\int_{\gamma} F f \psi d z=\int_{\gamma} F \psi \cdot f d z=0,
$$

for every $f \in \mathcal{A}(\mathbb{R})$. We have $F \in H(U \backslash K)$ and $\psi \in H_{0}\left(\mathbb{C}_{\infty} \backslash L\right)$, hence $F \psi \in H(U \backslash(K \cup L))$. It follows from Lemma 4.4 that there exists a function $a \in H(U)$ such that

$$
(F \psi)(z)=\left(\mathcal{E} F_{0} \psi\right)(z)=a(z)
$$

for $z \in U \backslash(K \cup L)$. For $z \in U \backslash(K \cup L)$ it holds that

$$
(F \psi)(z)=F(z) \psi(z)=\mathcal{E}(z) F_{0}(z) \psi(z) .
$$

For such points $z$ we may write

$$
\prod_{z_{n} \in(K \cup L)} E_{p_{n}}^{m_{n}}\left(\frac{z}{z_{n}}\right) \cdot \prod_{z_{n} \in(K \cup L)^{c}} E_{p_{n}}^{m_{n}}\left(\frac{z}{z_{n}}\right) \cdot F_{0}(z) \psi(z)=a(z) .
$$

This means that $a(z)$ vanishes for $z_{n} \in(K \cup L)^{c}$. Hence, for a different function $a \in H(U)$ we have

$$
\prod_{z_{n} \in(K \cup L)} E_{p_{n}}^{m_{n}}\left(\frac{z}{z_{n}}\right) \cdot F_{0}(z) \cdot \psi(z)=a(z)
$$

for $z \in U \backslash(K \cup L)$. For $z \in U \backslash(K \cup L)$ we can therefore write

$$
\psi(z)=\frac{1}{\prod_{z_{n} \in(K \cup L)} E_{p_{n}}^{m_{n}}\left(\frac{z}{z_{n}}\right)} \frac{a(z)}{F_{0}(z)},
$$


since $F_{0}$ does not vanish in $U \backslash K$. Note that other cancellations are still possible in the formula above. Nonetheless, representation (32) holds true. Choose now $N$ large enough to guarantee that

$$
\left\{n: z_{n} \in(K \cup L)\right\} \subset\{1, \ldots, N\} .
$$

Consider the symbol function

$$
G:=\prod_{n=1}^{N} E_{p_{n}}^{m_{n}}\left(\frac{z}{z_{n}}\right) \cdot F_{0}(z) .
$$

For any $f \in \mathcal{A}(\mathbb{R})$ we have

$$
\begin{aligned}
\left\langle T_{G} f, \psi\right\rangle & =\int_{\gamma} \prod_{n=1}^{N} E_{p_{n}}^{m_{n}}\left(\frac{z}{z_{n}}\right) F_{0}(z) f(z) \frac{1}{\prod_{z_{n} \in(K \cup L)} E_{p_{n}}^{m_{n}}\left(\frac{z}{z_{n}}\right)} \frac{a(z)}{F_{0}(z)} d z \\
& =\int_{\gamma} \frac{\prod_{n=1}^{N} E_{p_{n}}^{m_{n}}\left(\frac{z}{z_{n}}\right)}{\prod_{z_{n} \in(K \cup L)} E_{p_{n}}^{m_{n}}\left(\frac{z}{z_{n}}\right)} f(z) a(z) d z=0,
\end{aligned}
$$

by Cauchy's theorem. The curve $\gamma$ is a $C^{\infty}$ smooth Jordan curve with $K \cup L \subset$ $I(\gamma)$. It follows from Lemma 4.6 that

$$
\psi \in \operatorname{Span}\left\{\varphi, \frac{\varphi}{\left(z-z_{1}\right)}, \ldots, \frac{\varphi}{\left(z-z_{1}\right)^{m_{1}}}, \ldots, \frac{\varphi}{\left(z-z_{N}\right)}, \ldots, \frac{\varphi}{\left(z-z_{N}\right)^{m_{N}}}\right\} .
$$

This means that

$$
R\left(T_{F}\right)=\operatorname{ker} \varphi \cap \bigcap_{n=1}^{\infty} \bigcap_{k=1}^{m_{n}} \operatorname{ker} \frac{\varphi}{\left(z-z_{n}\right)^{k}}:=\mathfrak{K} .
$$

Indeed, assume that the equality does not hold. We showed in (31) that $R\left(T_{F}\right) \subset \mathfrak{K}$ and we also know from Theorem 2.2 that $R\left(T_{F}\right)$ is closed. Consider the locally convex space $\mathcal{A}(\mathbb{R}) / R\left(T_{F}\right)$. By the Hahn-Banach theorem there exists $\xi \in \mathcal{A}(\mathbb{R})^{\prime}$, which vanishes on $R\left(T_{F}\right)$ and $\xi(x) \neq 0$ for some $x \in \mathfrak{K} \backslash R\left(T_{F}\right)$. By (33) such a functional $\xi$ belongs to

$$
\operatorname{Span}\left\{\varphi, \frac{\varphi}{\left(z-z_{1}\right)}, \ldots, \frac{\varphi}{\left(z-z_{1}\right)^{m_{1}}}, \ldots, \frac{\varphi}{\left(z-z_{N}\right)}, \ldots, \frac{\varphi}{\left(z-z_{N}\right)^{m_{N}}}\right\}
$$

for some $N \in \mathbb{N}$. Hence it vanishes on $\mathfrak{K}$, which is a contradiction. We infer that $R\left(T_{F}\right)=\mathfrak{K}$. This proves the first assertion of the proposition.

Consider now the case index $T_{F_{0}}=k \geq 0$. Let now $\xi_{\psi}$ vanish on $R\left(T_{F}\right)$, i.e.

$$
\left\langle T_{F} f, \xi_{\psi}\right\rangle=0
$$

for every $f \in \mathcal{A}(\mathbb{R})$. We again conclude that there exists $a \in H(U)$ such that

$$
\mathcal{E}(z) \cdot F_{0}(z) \cdot \psi(z)=a(z)
$$

for $z \in U \backslash(K \cup L)$ and, as a result,

$$
\psi(z)=\frac{1}{\prod_{z_{n} \in(K \cup L)} E_{p_{n}}^{m_{n}}\left(\frac{z}{z_{n}}\right)} \frac{a(z)}{F_{0}(z)}
$$


for $z \in U \backslash(K \cup L)$. Choose $N \geq l$ large enough to guarantee that

$$
\left\{n: z_{n} \in K \cup L\right\} \subset\{1, \ldots, N\} .
$$

Consider the symbol

$$
H(z):=\prod_{n=1}^{N} E_{p_{n}}^{m_{n}}\left(\frac{z}{z_{n}}\right) \cdot F_{0}(z)
$$

We have

$$
\left\langle T_{H} f, \xi_{\psi}\right\rangle=\int_{\gamma} \frac{\prod_{n=1}^{N} E_{p_{n}}^{m_{n}}\left(\frac{z}{z_{n}}\right)}{\prod_{z_{n} \in K \cup L} E_{p_{n}}^{m_{n}}\left(\frac{z}{z_{n}}\right)} F_{0}(z) f(z) \frac{a(z)}{F_{0}(z)} d z=0,
$$

by Cauchy's theorem, since the integrand is holomorphic in $W \subset U \cap V$. It follows from Lemma 4.7 that

$$
\begin{gathered}
\psi \in \operatorname{Span}\left\{\varphi, \frac{\varphi}{\left(z-z_{l}\right)}, \ldots, \frac{\varphi}{\left(z-z_{l}\right)^{m_{l}-\mu_{l}}}, \frac{\varphi}{\left(z-z_{l+1}\right)}, \ldots, \frac{\varphi}{\left(z-z_{l+1}\right)^{m_{l+1}}},\right. \\
\left.\frac{\varphi}{\left(z-z_{N}\right)}, \ldots, \frac{\varphi}{\left(z-z_{N}\right)^{m_{N}}}\right\}
\end{gathered}
$$

As in the first part of the proof, it follows from the Hahn-Banach theorem that

$$
R\left(T_{F}\right)=\operatorname{ker} \varphi \cap \operatorname{ker} \frac{\varphi}{z-z_{l}} \cap \cdots \cap \operatorname{ker} \frac{\varphi}{\left(z-z_{l}\right)^{m_{l}-\mu_{l}}} \cap \bigcap_{i=l+1}^{\infty} \bigcap_{j=1}^{m_{i}} \operatorname{ker} \frac{\varphi}{\left(z-z_{i}\right)^{j}} .
$$

Now we consider the case index $F_{0}=k<-1$. This time the proof follows from Lemma 4.8.

We now prove representations (21) and (22). Assume first that

$$
\operatorname{index} T_{F_{0}}=-1 \text {. }
$$

Then, as in (30),

$$
R\left(T_{F_{0}}\right)=\operatorname{ker} \varphi
$$

which implies that

$$
\int_{\gamma} F_{0}(z) f(z) \varphi(z) d z=0
$$

for any $f \in \mathcal{A}(\mathbb{R})$. Hence,

$$
F_{0}(z) \varphi(z)=a(z)
$$

for some $a \in H(U)$ and $z \in U \backslash(K \cup L)$. Since $F_{0}$ does not vanish, we have

$$
\varphi(z)=\frac{a(z)}{F_{0}(z)}
$$

for $z \in U \backslash(K \cup L)$.

Assume that index $T_{F_{0}}=k<-1$. The function $\varphi$ is now chosen in such a way that

$$
\left\langle T_{G} f, \varphi\right\rangle=0
$$


for $f \in \mathcal{A}(\mathbb{R})$, where, let us recall,

$$
G(z)=\frac{F_{0}(z)}{\prod_{n=1}^{l-1} E_{p_{n}}^{m_{n}}\left(\frac{z}{z_{n}}\right) \cdot E_{p_{l}}^{\mu_{l}}\left(\frac{z}{z_{l}}\right)} .
$$

The numbers $l$ and $\mu_{l}$ were chosen in (27). For some $a \in H(U)$ we have

$$
\frac{F_{0}(z)}{\prod_{n=1}^{l-1} E_{p_{n}}^{m_{n}}\left(\frac{z}{z_{n}}\right) \cdot E_{p_{l}}^{\mu_{l}}\left(\frac{z}{z_{l}}\right)} \cdot \varphi(z)=a(z)
$$

for $z \in U \backslash(K \cup L), z \neq z_{1}, \ldots, z_{l}$. Hence, similarly as before,

$$
\varphi(z)=\prod_{n=1}^{l-1} E_{p_{n}}^{m_{n}}\left(\frac{z}{z_{n}}\right) \cdot E_{p_{l}}^{\mu_{l}}\left(\frac{z}{z_{l}}\right) \cdot \frac{a(z)}{F_{0}(z)} .
$$

The equality extends to $U \backslash(K \cup L)$. We therefore also have the representation

$$
\varphi(z)=\frac{a(z)}{F_{0}(z)}
$$

for some $a \in H(U)$.

Assume now that index $T_{F_{0}}=k \geq 0$. The function $\varphi$ is now chosen in such a way that

$$
\left\langle T_{G} f, \varphi\right\rangle=0
$$

for $f \in \mathcal{A}(\mathbb{R})$, where this time $G$ is equal to

$$
G(z)=\prod_{n=1}^{l-1} E_{p_{n}}^{m_{n}}\left(\frac{z}{z_{n}}\right) E_{p_{l}}^{\mu_{l}}\left(\frac{z}{z_{l}}\right) F_{0}(z) .
$$

The numbers $l$ and $\mu_{l}$ are chosen in (24). We therefore have

$$
\prod_{n=1}^{l-1} E_{p_{n}}^{m_{n}}\left(\frac{z}{z_{n}}\right) E_{p_{l}}^{\mu_{l}}\left(\frac{z}{z_{l}}\right) F_{0}(z) \cdot \varphi(z)=a(z)
$$

for some $a \in H(U)$ and $z \in U \backslash(K \cup L)$. Hence for $z \in U \backslash(K \cup L)$ and $z \neq z_{1}, \ldots, z_{l}$

$$
\varphi(z)=\frac{1}{\prod_{n=1}^{l-1} E_{p_{n}}^{m_{n}}\left(\frac{z}{z_{n}}\right) E_{p_{l}}^{\mu_{l}}\left(\frac{z}{z_{l}}\right)} \frac{a(z)}{F_{0}(z)} .
$$

Recall that we are guided by the proof of Theorem 4.1. This is why our next goal is a construction of dual functions in each case singled out in Proposition 4.3, i.e. functions $f_{i} \in \mathcal{A}(\mathbb{R}), i \in \mathbb{N}$ such that $\xi_{j}\left(f_{i}\right)=\delta_{i j}$. By $\xi_{j}, j \in \mathbb{N}$ we denoted the functionals for which it holds that

$$
R\left(T_{F}\right)=\bigcap_{j=1}^{\infty} \operatorname{ker} \xi_{j}
$$

We treat all three cases together. Observe that in each case there are $j \in \mathbb{N}$ and numbers $\nu_{n} \in \mathbb{N}$ such that

$$
R\left(T_{F}\right)=\operatorname{ker} \varphi \cap \bigcap_{n=j}^{\infty} \bigcap_{k=1}^{\nu_{n}} \operatorname{ker} \frac{\varphi}{\left(z-z_{n}\right)^{k}} .
$$


For simplicity we assume that $j=1$ and $\nu_{n}=m_{n}$ for $n \in \mathbb{N}$. either

The first step is of a different nature. Namely, there are two possibilities:

$$
\operatorname{ker} \varphi \cap \bigcap_{n=1}^{\infty} \bigcap_{k=1}^{m_{n}} \operatorname{ker} \frac{\varphi}{\left(z-z_{n}\right)^{k}}=\bigcap_{n=1}^{\infty} \bigcap_{k=1}^{m_{n}} \operatorname{ker} \frac{\varphi}{\left(z-z_{n}\right)^{k}},
$$

or the equality does not hold. In the latter case there exists a non-zero function $f \in \mathcal{A}(\mathbb{R})$ with

$$
f \in \bigcap_{n=1}^{\infty} \bigcap_{k=1}^{m_{n}} \operatorname{ker} \frac{\varphi}{\left(z-z_{n}\right)^{k}} \backslash\left(\operatorname{ker} \varphi \cap \bigcap_{n=1}^{\infty} \bigcap_{k=1}^{m_{n}} \operatorname{ker} \frac{\varphi}{\left(z-z_{n}\right)^{k}}\right) .
$$

The function $f / \xi_{\varphi}(f)$ is the first element of the construction in this case. Otherwise it suffices to construct functions $f \in \mathcal{A}(\mathbb{R})$ dual to functionals of the form $\frac{\varphi}{\left(z-z_{n}\right)^{k}}, n \in \mathbb{N}, 1 \leq k \leq m_{n}$, which is also the second step in the second situation.

So assume that $\xi$ is defined by a function $\frac{\varphi}{\left(z-z_{n}\right)^{k}}$ for some $n \in \mathbb{N}$ and $1 \leq k \leq m_{n}$ with $\varphi$ of the form postulated by Proposition 4.3 . We seek for a function $f \in \mathcal{A}(\mathbb{R})$ such that

$$
\begin{aligned}
\langle f, \varphi\rangle & =0, \\
\left\langle f, \frac{\varphi}{\left(z-z_{i}\right)^{j}}\right\rangle & =0
\end{aligned}
$$

for $i \neq n$ and $1 \leq j \leq m_{i}$ and also

$$
\left\langle f, \frac{\varphi}{\left(z-z_{n}\right)^{j}}\right\rangle=0
$$

for $j \neq k$. Such a function will be constructed by means of Toeplitz operators with appropriately chosen symbols. Let us recall that either

$$
\varphi=\frac{a}{F_{0}}
$$

for some function $a \in H(U)$ or

$$
\varphi(z)=\frac{1}{\prod_{n=1}^{l-1} E_{p_{n}}^{m_{n}}\left(\frac{z}{z_{n}}\right) E_{p_{l}}^{\mu_{l}}\left(\frac{z}{z_{l}}\right)} \frac{a(z)}{F_{0}(z)} .
$$

Representation (36) holds when index $T_{F_{0}} \leq-1$, while (37) if index $T_{F_{0}} \geq 0$. Our consideration must be subordinate to these two cases.

Denote

$$
A:=\left\{n \in \mathbb{N}: a\left(z_{n}\right)=0\right\} .
$$

Observe that $A$ is finite. This follows from (36) and (37) since $a(z)=0$ implies $\varphi(z)=0$. Recall that $\varphi \in H_{0}\left(\mathbb{C}_{\infty} \backslash L\right)$. Thus zeros of $\varphi$ cannot accumulate at $\pm \infty$. For $n \in A$ let $\alpha_{n}$ be the multiplicity of $z_{n}$. Write

$$
a(z)=\prod_{n \in A}\left(z-z_{n}\right)^{\alpha_{n}} b(z)
$$

for a function $b \in H(U)$. 
Assume first that index $T_{F_{0}} \leq-1$. Consider the symbol function

$$
F_{n}(z):=\frac{\prod_{i \neq n} E_{p_{i}}^{m_{i}}\left(\frac{z}{z_{i}}\right)}{\prod_{i \in A}\left(z-z_{i}\right)^{\alpha_{i}}} \cdot F_{0}(z) .
$$

Observe that $F_{n}$ is indeed a symbol function, since as we noted, $A$ is finite. Let now $g \in \mathcal{A}(\mathbb{R})$ be arbitrary. For $\iota \neq n$ and $1 \leq \kappa \leq m_{\iota}$ it holds that

$$
\begin{aligned}
\left\langle T_{F_{n}} g, \frac{\varphi}{\left(z-z_{\iota}\right)^{\kappa}}\right\rangle & =\int_{\gamma} F_{n}(z) g(z) \frac{\varphi(z)}{\left(z-z_{\iota}\right)^{\kappa}} d z \\
& =\int_{\gamma} \frac{\prod_{i \neq n} E_{p_{i}}^{m_{i}}\left(\frac{z}{z_{i}}\right)}{\prod_{i \in A}\left(z-z_{i}\right)^{\alpha_{i}}} \cdot F_{0}(z) \cdot \frac{1}{\left(z-z_{\iota}\right)^{\kappa}} \cdot g(z) \cdot \frac{\prod_{i \in A}\left(z-z_{i}\right)^{\alpha_{i}} b(z)}{F_{0}(z)} d z \\
& =\int_{\gamma} \frac{\prod_{i \neq n} E_{p_{i}}^{m_{i}}\left(\frac{z}{z_{i}}\right)}{\left(z-z_{\iota}\right)^{\kappa}} \cdot g(z) \cdot b(z) d z=0,
\end{aligned}
$$

by Cauchy's theorem, since the integrand is holomorphic. We used Proposition 4.3 , formula (21). If index $T_{F_{0}} \geq 0$ we set

$$
\mathcal{F}_{l}(z):=\frac{\prod_{i \neq l} E_{p_{i}}^{m_{i}}\left(\frac{z}{z_{i}}\right) E_{p_{l}}^{\mu_{l}}\left(\frac{z}{z_{l}}\right)}{\prod_{i \in A}\left(z-z_{i}\right)^{\alpha_{i}}} \cdot F_{0}(z) .
$$

and

$$
\mathcal{F}_{n}(z):=\frac{\prod_{i \neq n} E_{p_{i}}^{m_{i}}\left(\frac{z}{z_{i}}\right)}{\prod_{i \in A}\left(z-z_{i}\right)^{\alpha_{i}}} \cdot F_{0}(z)
$$

for $n>l$. The number $l$ was chosen in (24). It follows from Proposition 4.3 that if index $T_{F_{0}} \geq 0$, then we need to find functions dual to the functions

$$
\frac{\varphi}{\left(z-z_{l}\right)}, \ldots, \frac{\varphi}{\left(z-z_{l}\right)^{m_{l}-\mu_{l}}}, \frac{\varphi}{\left(z-z_{l+1}\right)}, \ldots, \frac{\varphi}{\left(z-z_{l+1}\right)^{m_{l+1}}}, \ldots
$$

If $n>l$ and $\iota \neq n$ then for every $g \in \mathcal{A}(\mathbb{R})$ we have,

$$
\begin{aligned}
\left\langle T_{\mathcal{F}_{n}} g, \frac{\varphi}{\left(z-z_{\iota}\right)^{\kappa}}\right\rangle= & \int_{\gamma} \frac{\prod_{i \neq n} E_{p_{i}}^{m_{i}}\left(\frac{z}{z_{i}}\right)}{\prod_{i \in A}\left(z-z_{i}\right)^{\alpha_{i}}} \cdot F_{0}(z) \cdot \frac{1}{\left(z-z_{\iota}\right)^{\kappa}} \cdot g(z) \\
& \cdot \frac{\prod_{i \in A}\left(z-z_{i}\right)^{\alpha_{i}} b(z)}{\prod_{n=1}^{l-1} E_{p_{n}}^{m_{n}}\left(\frac{z}{z_{n}}\right) E_{p_{l}}^{\mu_{l}}\left(\frac{z}{z_{l}}\right) \cdot F_{0}(z)} d z
\end{aligned}
$$

by Proposition 4.3. Observe that either $\iota>l$ or $\iota=l$ and $\kappa+\mu_{l} \leq m_{l}$. We infer that also in this situation

$$
\left\langle T_{\mathcal{F}_{n}} g, \frac{\varphi}{\left(z-z_{\iota}\right)^{\kappa}}\right\rangle=0 .
$$


Consider now the case $n=l$. Then $\iota>n=l$ and we have

$$
\begin{aligned}
\left\langle T_{\mathcal{F}_{l}} g, \frac{\varphi}{\left(z-z_{\iota}\right)^{\kappa}}\right\rangle= & \int_{\gamma} \frac{\prod_{i \neq l} E_{p_{i}}^{m_{i}}\left(\frac{z}{z_{i}}\right)}{\prod_{i \in A}\left(z-z_{i}\right)^{\alpha_{i}}} \cdot F_{0}(z) \cdot \frac{E_{p_{l}}^{\mu_{l}}\left(\frac{z}{z_{l}}\right)}{\left(z-z_{\iota}\right)^{\kappa}} \cdot g(z) \\
& \cdot \frac{\prod_{i \in A}\left(z-z_{i}\right)^{\alpha_{i}} b(z)}{\prod_{n=1}^{l-1} E_{p_{n}}^{m_{n}}\left(\frac{z}{z_{n}}\right) E_{p_{l}}^{\mu_{l}}\left(\frac{z}{z_{l}}\right) \cdot F_{0}(z)} d z \\
= & \int_{\gamma} \prod_{i>l} E_{p_{i}}^{m_{i}}\left(\frac{z}{z_{i}}\right) \frac{1}{\left(z-z_{\iota}\right)^{\kappa}} \cdot g(z) \cdot b(z) d z=0 .
\end{aligned}
$$

Observe that in every case considered above we have $\left\langle T_{F_{n}} g, \varphi\right\rangle=0$ (resp. $\left.\left\langle T_{\mathcal{F}_{l}} g, \varphi\right\rangle=0,\left\langle T_{\mathcal{F}_{n}} g, \varphi\right\rangle=0\right)$ for every $g \in \mathcal{A}(\mathbb{R})$. This means that functions of the form $T_{F_{n}} g, g \in \mathcal{A}(\mathbb{R})$ (resp. $T_{\mathcal{F}_{l}} g, T_{\mathcal{F}_{n}} g$ ) are good candidates for $f$ in formulas (34). Indeed, for every $g \in \mathcal{A}(\mathbb{R})$ conditions (34) are satisfied. In order to have dual functions we need therefore to choose functions $g_{n, k} \in$ $\mathcal{A}(\mathbb{R}), n \in \mathbb{N}, k=1, \ldots, m_{n}$ such that

$$
\left\langle T_{F_{n}}\left(g_{n, k}\right), \frac{\varphi}{\left(z-z_{n}\right)^{j}}\right\rangle=\delta_{k j}, j=1, \ldots, m_{n},
$$

when index $T_{F_{0}} \leq-1$. If index $T_{F_{0}} \geq 0$ we have $n>l$ or $n=l$ and then $j, k=1, \ldots, m_{l}-\mu_{l}$.

We treat the case index $T_{F_{0}} \leq-1$ first. By Cauchy's integral formula

$$
\begin{aligned}
\left\langle T_{F_{n}} g, \frac{\varphi}{\left(z-z_{n}\right)^{j}}\right\rangle & =\int_{\gamma} \frac{\prod_{i \neq n} E_{p_{i}}^{m_{i}}\left(\frac{z}{z_{i}}\right) \cdot b(z) \cdot g(z)}{\left(z-z_{n}\right)^{j}} d z \\
& =\frac{2 \pi i}{(j-1) !} \frac{d^{j-1}}{d z^{j-1}}\left(\prod_{i \neq n} E_{p_{i}}^{m_{i}}\left(\frac{z}{z_{i}}\right) \cdot b(z) \cdot g(z)\right)\left(z_{n}\right) .
\end{aligned}
$$

Thus the function $g_{n, k} \in \mathcal{A}(\mathbb{R})$ must be chosen in such a way that

$$
\frac{2 \pi i}{(j-1) !} \frac{d^{j-1}}{d z^{j-1}}\left(\prod_{i \neq n} E_{p_{i}}^{m_{i}}\left(\frac{z}{z_{i}}\right) \cdot b(z) \cdot g_{n, k}(z)\right)\left(z_{n}\right)=\delta_{k j},
$$

$j=1, \ldots, m_{n}$. System of equations (38) is easily solvable inductively for$g_{n, k}^{(j)}\left(z_{n}\right), j=1, \ldots, m_{n}$, since both $b(z)$ and $\prod_{i \neq n} E_{p_{i}}^{m_{i}}\left(\frac{z}{z_{i}}\right)$ do not vanish at $z_{n}$. Obviously for $g_{n, k}$ one can take an appropriate interpolating polynomial.

Assume now that index $T_{F_{0}} \geq 0$ and $n>l$. Then

$$
\begin{aligned}
\left\langle T_{\mathcal{F}_{n}} g, \frac{\varphi}{\left(z-z_{n}\right)^{j}}\right\rangle & =\int_{\gamma} \frac{\prod_{i \neq n} E_{p_{i}}^{m_{i}}\left(\frac{z}{z_{i}}\right) \cdot b(z) \cdot g(z)}{\prod_{i=1}^{l-1} E_{p_{i}}^{m_{i}}\left(\frac{z}{z_{i}}\right) E_{p_{l}}^{\mu_{l}}\left(\frac{z}{z_{l}}\right) \cdot\left(z-z_{n}\right)^{j}} d z \\
& =\frac{2 \pi i}{(j-1) !} \frac{d^{j-1}}{d z^{j-1}}\left(E_{p_{l}}^{m_{l}-\mu_{l}}\left(\frac{z}{z_{l}}\right) \cdot \prod_{i \neq n, i>l} E_{p_{i}}^{m_{i}}\left(\frac{z}{z_{i}}\right) \cdot b(z) \cdot g(z)\right)\left(z_{n}\right) .
\end{aligned}
$$

Again we seek for functions $g_{n, k}, k=1, \ldots, m_{n}$ such that

$$
\frac{2 \pi i}{(j-1) !} \frac{d^{j-1}}{d z^{j-1}}\left(E_{p_{l}}^{m_{l}-\mu_{l}}\left(\frac{z}{z_{l}}\right) \cdot \prod_{i \neq n, i>l} E_{p_{i}}^{m_{i}}\left(\frac{z}{z_{i}}\right) \cdot b(z) \cdot g_{n, k}(z)\right)\left(z_{n}\right)=\delta_{k j},
$$

$j=1, \ldots, m_{n}$. This system of equations is again easily solvable for $g_{n, k}^{(j)}\left(z_{n}\right)$. 
Let now index $T_{F_{0}} \geq 0$ and $n=l$. Under these assumptions we have also that $j=1, \ldots, m_{l}-\mu_{l}$. Then

$$
\begin{aligned}
\left\langle T_{\mathcal{F}_{l}} g, \frac{\varphi}{\left(z-z_{l}\right)^{j}}\right\rangle & =\int_{\gamma} \frac{\prod_{i \neq l} E_{p_{i}}^{m_{i}}\left(\frac{z}{z_{i}}\right) \cdot E_{p_{l}}^{\mu_{l}}\left(\frac{z}{z_{l}}\right) \cdot b(z) \cdot g(z)}{\prod_{i=1}^{l-1} E_{p_{i}}^{m_{i}}\left(\frac{z}{z_{i}}\right) E_{p_{l}}^{\mu_{l}}\left(\frac{z}{z_{l}}\right) \cdot\left(z-z_{l}\right)^{j}} d z \\
& =\frac{2 \pi i}{(j-1) !} \frac{d^{j-1}}{d z^{j-1}}\left(\prod_{i>l} E_{p_{i}}^{m_{i}}\left(\frac{z}{z_{i}}\right) \cdot b(z) \cdot g(z)\right)\left(z_{l}\right) .
\end{aligned}
$$

Again it is easy to choose appropriate functions $g_{l, k}$. We proved the following:

Proposition 4.9. Assume that $F \in H(U \backslash K), U$ an open neighborhood of $\mathbb{R}$ and $K$ a compact subset of $\mathbb{R}$. Furthermore, assume that $F$ vanishes only at points $z_{n} \in \mathbb{R} \backslash K, n \in \mathbb{N}$ which accumulate only at $\pm \infty$. There exists functionals $\xi_{n} \in \mathcal{A}(\mathbb{R})^{\prime}$ and functions $f_{m} \in \mathcal{A}(\mathbb{R}), m, n \in \mathbb{N}$ such that

(i) $\xi_{n}\left(f_{m}\right)=\delta_{m n}$,

(ii) $R\left(T_{F}\right)=\bigcap \operatorname{ker} \xi_{n}$.

We are now in a position where the arguments of the proof of Theorem 4.1 can be applied to prove Theorem 3 .

Proof of Theorem 3. Assume that there exists a continuous projection

$$
P: \mathcal{A}(\mathbb{R}) \rightarrow \mathcal{A}(\mathbb{R})
$$

onto the range of the operator $T_{F}$. Denote

$$
X_{N}:=\left\{f \in \mathcal{A}: \xi_{N+1}(f)=\xi_{N+2}(f)=\cdots=0\right\},
$$

where

$$
R\left(T_{F}\right)=\bigcap_{n=1}^{\infty} \operatorname{ker} \xi_{n}
$$

The functionals $\xi_{n}, n \in \mathbb{N}$ were constructed in Proposition 4.3. Denote also

$$
X:=\bigcup_{N=1}^{\infty} X_{N}
$$

We stress that if

$$
\operatorname{ker} \varphi \cap \bigcap_{n=1}^{\infty} \bigcap_{k=1}^{m_{n}} \operatorname{ker} \frac{\varphi}{\left(z-z_{n}\right)^{k}}=\bigcap_{n=1}^{\infty} \bigcap_{k=1}^{m_{n}} \operatorname{ker} \frac{\varphi}{\left(z-z_{n}\right)^{k}},
$$

then the $\xi_{n}$ correspond only to the functions $\frac{\varphi}{\left(z-z_{n}\right)^{k}}$, not to the function $\varphi$ itself.

For $f \in X$ the expression

$$
f-\sum_{n=1}^{\infty} \xi_{n}(f) f_{n}
$$


is well-defined, since the sum is finite. The functions $f_{n}$, constructed in Proposition 4.9 , satisfy $\xi_{m}\left(f_{n}\right)=\delta_{m n}$. For any $m \in \mathbb{N}$ we have

$$
\xi_{m}\left(f-\sum_{n=1}^{\infty} \xi_{n}(f) f_{n}\right)=\xi_{m}(f)-\xi_{m}(f)=0 .
$$

Hence for every $f \in X$,

$$
f-\sum_{n=1}^{\infty} \xi_{n}(f) f_{n} \in R\left(T_{F}\right)
$$

by (39). Thus for $f \in X$,

$$
f-\sum_{n=1}^{\infty} \xi_{n}(f) f_{n}=P\left(f-\sum_{n=1}^{\infty} \xi_{n}(f) f_{n}\right) .
$$

In other words,

$$
(I-P) f=\sum_{n=1}^{\infty} \xi_{n}(f) \psi_{n}
$$

for $f \in X$, where $\psi_{n}=(I-P)\left(f_{n}\right)$. Observe that $f_{n} \neq P f_{n}$, since $\xi_{n}\left(f_{n}\right)=1$. This implies that none of the functions $\psi_{n}$ vanishes identically. There exists therefore $\tilde{z} \in \mathbb{R}$ such that $\psi_{n}(\tilde{z}) \neq 0$ for every $n \in \mathbb{N}$. We shall construct a sequence of functions $\phi_{n} \in X$ convergent in $\mathcal{A}(\mathbb{R})$ such that $(I-P) \phi_{n}$ cannot converge. This shows that the projection $P$ cannot exist.

Recall representations (21) and (22) from Proposition 4.3. We consider now those points $z_{n}$ which satisfy $a\left(z_{n}\right) \neq 0$. There exists an entire function $f$ such that

$$
\frac{2 \pi i}{j !}(a \cdot f)^{(j)}\left(z_{n}\right)=\frac{1}{\psi_{M}(\tilde{z})}, j=0, \ldots, m_{n}-1, n \in \mathbb{N},
$$

where $\psi_{M}=(I-P) f_{M}$ and $f_{M}$ is dual to $\xi_{M}=\frac{\varphi}{\left(z-z_{n}\right)^{j+1}}$. We have

$$
\begin{aligned}
(a \cdot f)^{(j)}\left(z_{n}\right) & =\sum_{i=0}^{j} \frac{j !}{i !(j-i) !} a^{(i)}\left(z_{n}\right) f^{(j-i)}\left(z_{n}\right) \\
& =a\left(z_{n}\right) f^{(j)}\left(z_{n}\right)+\sum_{i=1}^{j} \frac{j !}{i !(j-i) !} a^{(i)}\left(z_{n}\right) f^{(j-i)}\left(z_{n}\right)
\end{aligned}
$$

for $j=0, \ldots, m_{n}-1$. If $a\left(z_{n}\right) \neq 0$ system $(41)$ can be solved for $f^{(j)}\left(z_{n}\right)$. If $a\left(z_{n}\right)=0$ we take $f$ to satisfy $f\left(z_{n}\right)=f^{\prime}\left(z_{n}\right)=\cdots=f^{\left(m_{n}-1\right)}\left(z_{n}\right)=0$. Let us recall that $a\left(z_{n}\right)=0$ only for a finite number of $n \in \mathbb{N}$. Thus the entire function $f$ is chosen to satisfy equations (41) for all but a finite number of points $z_{n}$ at which it is assumed to vanish to degree $m_{n}-1$.

Assume first that index $T_{F_{0}} \leq-1$. Consider the symbol function

$$
F_{N}(z):=\prod_{n=N+1}^{\infty} E_{p_{n}}^{m_{n}}\left(\frac{z}{z_{n}}\right) \cdot F_{0}(z)
$$


At this moment the $p_{n}$ are only assumed to guarantee the convergence of the product. Under this assumption if $i>N$ and $1 \leq k \leq m_{i}$, then

$$
\left\langle T_{F_{N}} f, \frac{\varphi}{\left(z-z_{i}\right)^{k}}\right\rangle=\int_{\gamma} \frac{\prod_{n=N+1}^{\infty} E_{p_{n}}^{m_{n}}\left(\frac{z}{z_{n}}\right) \cdot F_{0}(z)}{\left(z-z_{i}\right)^{k}} \cdot f(z) \cdot \frac{a(z)}{F_{0}(z)} d z=0
$$

by Cauchy's theorem, since the integrand is holomorphic. This implies that $T_{F_{N}} f \in X$ for every $N \in \mathbb{N}$. We now choose $p_{n}$ so that

$$
(I-P)\left(T_{F_{N}} f\right)
$$

diverges at $\tilde{z}$. This gives a contradiction, since

$$
\prod_{n=N+1}^{\infty} E_{p_{n}}^{m_{n}}\left(\frac{z}{z_{n}}\right) \cdot f(z)
$$

converges uniformly on compact subsets of the plane to $f$ as $N \rightarrow \infty$. Hence the restriction of this function to the real line converges to $\left.f\right|_{\mathbb{R}}$ in $\mathcal{A}(\mathbb{R})$. Since the operator $T_{F_{0}}$ is continuous,

$$
T_{F_{N}} f=T_{F_{0}}\left(\prod_{n=N+1}^{\infty} E_{p_{n}}^{m_{n}}\left(\frac{z}{z_{n}}\right) \cdot f(z)\right) \rightarrow T_{F_{0}} f
$$

in $\mathcal{A}(\mathbb{R})$. Thus if $P$ is a continuous operator we should have

$$
(I-P)\left(T_{F_{N}} f\right) \rightarrow(I-P)\left(T_{F_{0}} f\right)
$$

and, since the functionals of evaluation at points of $\mathbb{R}$ are continuous on $\mathcal{A}(\mathbb{R})$, also

$$
(I-P)\left(T_{F_{N}} f\right)(\tilde{z}) \rightarrow(I-P)\left(T_{F_{0}} f\right)(\tilde{z}) .
$$

We shall show that this is impossible by appropriately choosing the numbers $p_{n}$, actually $p_{n}^{N}$, since the sequence will be different for each $N$. Thus

$$
F_{N}(z)=\prod_{n=N+1}^{\infty} E_{p_{n}^{N}}^{m_{n}}\left(\frac{z}{z_{n}}\right) \cdot F_{0}(z) .
$$

For $1 \leq i \leq N$ and $2 \leq j \leq m_{i}$, provided $m_{i} \geq 2$, we have

$$
\begin{aligned}
\left\langle T_{F_{N}} f, \frac{\varphi}{\left(z-z_{i}\right)^{j}}\right\rangle= & \int_{\gamma} \frac{\prod_{n=N+1}^{\infty} E_{p_{n}^{N}}^{m_{n}}\left(\frac{z}{z_{n}}\right) \cdot f(z) \cdot a(z)}{\left(z-z_{i}\right)^{j}} d z \\
= & \frac{2 \pi i}{(j-1) !} \frac{d^{j-1}}{d z^{j-1}}\left(\prod_{n=N+1}^{\infty} E_{p_{n}^{N}}^{m_{n}}\left(\frac{z}{z_{n}}\right) \cdot f(z) \cdot a(z)\right)\left(z_{i}\right) \\
= & \frac{2 \pi i}{(j-1) !} \sum_{k=0}^{j-1} \frac{(j-1) !}{k !(j-1-k) !} \\
& \left(\prod_{n=N+1}^{\infty} E_{p_{n}^{N}}^{m_{n}}\left(\frac{z}{z_{n}}\right)\right)^{(k)}\left(z_{i}\right)(f \cdot a)^{(j-1-k)}\left(z_{i}\right) .
\end{aligned}
$$


Thus for $1 \leq i \leq N$ and $2 \leq j \leq m_{i}$,

$$
\begin{aligned}
\left\langle T_{F_{N}} f, \frac{\varphi}{\left(z-z_{i}\right)^{j}}\right\rangle= & \frac{2 \pi i}{(j-1) !} \prod_{n=N+1}^{\infty} E_{p_{n}^{N}}^{m_{n}}\left(\frac{z_{i}}{z_{n}}\right) \cdot(f \cdot a)^{(j-1)}\left(z_{i}\right) \\
& +\frac{2 \pi i}{(j-1) !} \sum_{k=1}^{j-1} \frac{(j-1) !}{k !(j-1-k) !} \\
& \left(\prod_{n=N+1}^{\infty} E_{p_{n}^{N}}^{m_{n}}\left(\frac{z_{i}}{z_{n}}\right)\right)^{(k)}\left(z_{i}\right)(f \cdot a)^{(j-1-k)}\left(z_{i}\right) .
\end{aligned}
$$

Obviously, if $j=1$ formula (42) reduces just to

$$
\left\langle T_{F_{N}} f, \frac{\varphi}{z-z_{i}}\right\rangle=2 \pi i \prod_{n=N+1}^{\infty} E_{p_{n}^{N}}^{m_{n}}\left(\frac{z_{i}}{z_{n}}\right) \cdot f\left(z_{i}\right) \cdot a\left(z_{i}\right) .
$$

Roughly speaking the rest of a convergent product converges uniformly on compact subsets of $\mathbb{C}$ to 1 , its derivatives to 0 . Hence for large $N$

$$
\left\langle T_{F_{N}} f, \frac{\varphi}{\left(z-z_{i}\right)^{j}}\right\rangle \sim \frac{2 \pi i}{(j-1) !}(f \cdot a)^{j-1}\left(z_{i}\right)=\frac{1}{\psi_{M}(\tilde{z})},
$$

where as before $\psi_{M}=(I-P) f_{M}$ with $f_{M}$ dual to $\xi_{M}=\frac{\varphi}{\left(z-z_{i}\right)^{j}}$. Consequently,

$$
\left\langle T_{F_{N}} f, \frac{\varphi}{\left(z-z_{i}\right)^{j}}\right\rangle \psi_{M}(\tilde{z}) \sim 1
$$

From now on we consider only those natural numbers $N \in \mathbb{N}$ which satisfy $\left|z_{N}\right|<\left|z_{N+1}\right|$. It follows from (19) that these numbers constitute a subsequence of $\mathbb{N}$. For such a number $N \in \mathbb{N}$ we choose numbers $p_{n}^{N}, n>N$ such that

$$
\left|\prod_{n=N+1}^{\infty} E_{p_{n}^{N}}^{m_{n}}\left(\frac{z}{z_{n}}\right)-1\right| \leq \frac{1}{3} \varepsilon_{N}
$$

for $|z| \leq\left|z_{N}\right|+\delta_{N}$, where the numbers $\delta_{N}>0$ are chosen in such a way that $\left|z_{N}\right|+\bar{\delta}_{N}<\left|z_{N+1}\right|$. We first determine the numbers $\varepsilon_{N}>0$ and then show that appropriate sequences $\left(p_{n}^{N}\right)$ can also be chosen. Denote

$$
S_{N}(z):=\prod_{n=N+1}^{\infty} E_{p_{n}^{N}}^{m_{n}}\left(\frac{z}{z_{n}}\right) .
$$

By Cauchy's integral formula for $k>0$

$$
S_{N}^{(k)}(z):=\frac{k !}{2 \pi i} \int_{|\zeta|=\left|z_{N}\right|+\delta_{N}} \frac{\left(S_{N}(\zeta)-1\right)}{(\zeta-z)^{k+1}} d \zeta
$$

for $|z|<\left|z_{N}\right|+\delta_{N}$, in particular for $z=z_{1}, \ldots, z_{N}$. Hence for $|z| \leq\left|z_{N}\right|$,

$$
\left|S_{N}^{(k)}(z)\right| \leq k ! \frac{\max _{|\zeta| \leq\left|z_{N}\right|+\delta_{N}}\left|S_{N}(\zeta)-1\right|}{\delta_{N}^{k+1}}\left(\left|z_{N}\right|+\delta_{N}\right) \leq k ! \frac{\varepsilon_{N}\left(\left|z_{N}\right|+\delta_{N}\right)}{3 \delta_{N}^{k+1}}
$$


if $\varepsilon_{N}$ satisfies (44). For any $i=1, \ldots, N$ such that $m_{i} \geq 2$ the number $\epsilon_{N}>0$ must therefore be chosen in such a way that $\varepsilon_{N}<1$ and

$$
\frac{2 \pi}{(j-1) !} \sum_{k=1}^{j-1} \frac{(j-1) ! k !}{k !(j-1-k) !} \frac{\varepsilon_{N}\left(\left|z_{N}\right|+\delta_{N}\right)}{3 \delta_{N}^{k+1}}\left|(f \cdot a)^{(j-1-k)}\left(z_{i}\right)\right| \cdot\left|\psi_{M}(\tilde{z})\right| \leq \frac{1}{3},
$$

for $j=2, \ldots, m_{i}$, where $\psi_{M}$ corresponds to $\frac{\varphi}{\left(z-z_{i}\right)^{j}}$. This guarantees that the second term in (43) is uniformly small. If $m_{i}=1$, then this condition is void. In particular, if $m_{1}=\cdots=m_{N}=1$, then one can simply take $\varepsilon_{N}=1$. In general, for a fixed number $N$ the number $\varepsilon_{N}$ must satisfy a finite number of conditions (45) corresponding to $i=1, \ldots, N$ and $j=2, \ldots, m_{i}$. Thus such a number can be chosen.

Let $\xi_{M}=\frac{\varphi}{\left(z-z_{i}\right)^{j}}$ with $i \leq N, 2 \leq j \leq m_{i}$. Let also $\psi_{M}=(I-P) f_{M}$, where $f_{M}$ is dual to $\xi_{M}$. With such a choice of $\varepsilon_{N}$, under the assumption that $a\left(z_{n}\right) \neq 0$, we have

$$
\begin{aligned}
& \left|\left\langle T_{F_{N}} f, \xi_{M}\right\rangle \psi_{M}(\tilde{z})-1\right| \\
& \leq\left|\frac{2 \pi i}{(j-1) !} \prod_{n=N+1}^{\infty} E_{p_{n}^{N}}^{m_{n}}\left(\frac{z_{i}}{z_{n}}\right) \cdot(a \cdot f)^{(j-1)}\left(z_{i}\right) \cdot \psi_{M}(\tilde{z})-1\right| \\
& \quad+\frac{2 \pi}{(j-1) !} \sum_{k=1}^{j-1} \frac{(j-1) !}{k !(j-1-k) !}\left|\left(\prod_{n=N+1}^{\infty} E_{p_{n}^{N}}^{m_{n}}\left(\frac{z_{i}}{z_{n}}\right)\right)^{(k)}\left(z_{i}\right)\right| \\
& \quad \cdot\left|(f \cdot a)^{(j-1-k)}\left(z_{i}\right)\right| \cdot\left|\psi_{M}(\tilde{z})\right| \leq \frac{2}{3} .
\end{aligned}
$$

The second term is $\leq \frac{1}{3}$ by (45). From the choice of the function $f$ in (41) and (44) it follows that the first does not exceed $\frac{1}{3}$, as well. If $j=1$, then the estimate reduces just to (44). Thus, it follows from (40) that

$$
\left|(I-P)\left(T_{F_{N}} f\right)(\tilde{z})-\sum_{n=1}^{N} m_{n}\right| \leq \frac{2}{3} \sum_{n=1}^{N} m_{n},
$$

which shows that

$$
\left|(I-P)\left(T_{F_{N}} f\right)(\tilde{z})\right| \geq \sum_{n=1}^{N} m_{n}-\left|(I-P)\left(T_{F_{N}} f\right)(\tilde{z})-\sum_{n=1}^{N} m_{n}\right| \geq \frac{1}{3} \sum_{n=1}^{N} m_{n} \rightarrow \infty
$$

as $N \rightarrow \infty$. This estimate is valid under the assumption that $a$ does not vanish on any $z_{n}$. If this is not the case, the estimate differs by a constant. The conclusion is however the same, namely, $(I-P)\left(T_{F_{N}} f\right)(\tilde{z})$ diverges.

Thus to complete the proof we need to choose the numbers $p_{n}^{N}$ in such a way that (44) holds true. Fix $N$. There exists a number $\eta_{N}>0$ such that if $|z| \leq \eta_{N}$, then $\left|e^{z}-1\right| \leq \varepsilon_{N} / 3$. Also, 


$$
\begin{aligned}
\left|\sum_{n=N+1}^{\infty} \log E_{p_{n}^{N}}^{m_{n}}\left(\frac{z}{z_{n}}\right)\right| & \leq \sum_{n=N+1}^{\infty}\left|\log E_{p_{n}^{N}}^{m_{n}}\left(\frac{z}{z_{n}}\right)\right| \\
& \leq \frac{3}{2} \sum_{n=N+1}^{\infty} m_{n}\left|E_{p_{n}^{N}}\left(\frac{z}{z_{n}}\right)-1\right| \leq \frac{3}{2} \sum_{n=N+1}^{\infty} m_{n}\left|\frac{z}{z_{n}}\right|^{p_{n}^{N}+1} .
\end{aligned}
$$

It suffices therefore to choose the numbers $p_{n}^{N}$ in such a way that

$$
\frac{3}{2} \sum_{n=N+1}^{\infty} m_{n}\left(\frac{\left|z_{N}\right|+\delta_{N}}{\left|z_{n}\right|}\right)^{p_{n}^{N}+1} \leq \epsilon_{N}
$$

and that for any $r>0$ the series

$$
\sum_{n=N+1}^{\infty} m_{n}\left(\frac{r}{\left|z_{n}\right|}\right)^{p_{n}^{N}+1}
$$

converges. Such a choice is obviously possible and it guarantees inequality (44). We may assume that $p_{n}^{N} \leq p_{n}^{N+1}$. This implies that $\prod_{n=N+1}^{\infty} E_{p_{n}^{N}}^{m_{n}}$ converges in $H(\mathbb{C})$ to 1 as $N \rightarrow \infty$.

Assume now that index $T_{F_{0}} \geq 0$. Then we set

$$
F_{N}(z):=\prod_{n=1}^{l-1} E_{p_{n}}^{m_{n}}\left(\frac{z}{z_{n}}\right) \cdot E_{p_{l}}^{\mu_{l}}\left(\frac{z}{z_{l}}\right) \cdot \prod_{n=N+1}^{\infty} E_{p_{n}^{N}}^{m_{n}}\left(\frac{z}{z_{n}}\right) \cdot F_{0}(z)
$$

for $N \geq l$. For any $f \in \mathcal{A}(\mathbb{R})$ we have

$$
\begin{aligned}
& \left\langle T_{F_{N}} f, \frac{\varphi}{\left(z-z_{n}\right)^{k}}\right\rangle \\
& =\int_{\gamma} \frac{\prod_{n=1}^{l-1} E_{p_{n}}^{m_{n}}\left(\frac{z}{z_{n}}\right) \cdot E_{p_{l}}^{\mu_{l}}\left(\frac{z}{z_{l}}\right) \cdot \prod_{n=N+1}^{\infty} E_{p_{n}}^{m_{n}}\left(\frac{z}{z_{n}}\right) \cdot F_{0}(z)}{\prod_{n=1}^{l-1} E_{p_{n}}^{m_{n}}\left(\frac{z}{z_{n}}\right) \cdot E_{p_{l}}^{\mu_{l}}\left(\frac{z}{z_{l}}\right)\left(z-z_{n}\right)^{k}} \\
& \quad \cdot f(z) \cdot \frac{a(z)}{F_{0}(z)} d z=0
\end{aligned}
$$

for $n>N$ and $k=1, \ldots, m_{n}$. We used representation (22) of $\varphi$ in Proposition 4.3. Hence $T_{F_{N}} f \in X$ for any $f \in \mathcal{A}(\mathbb{R})$. For $l \leq i \leq N$ and $1 \leq j \leq m_{i}$ we have

$$
\begin{aligned}
& \left\langle T_{F_{N}} f, \frac{\varphi}{\left(z-z_{i}\right)^{j}}\right\rangle \\
& =\int_{\gamma} \frac{\prod_{n=1}^{l-1} E_{p_{n}}^{m_{n}}\left(\frac{z}{z_{n}}\right) \cdot E_{p_{l}}^{\mu_{l}}\left(\frac{z}{z_{l}}\right) \cdot \prod_{n=N+1}^{\infty} E_{p_{n}^{N}}^{m_{n}}\left(\frac{z}{z_{n}}\right) \cdot F_{0}(z) \cdot a(z)}{\prod_{n=1}^{l-1} E_{p_{n}}^{m_{n}}\left(\frac{z}{z_{n}}\right) \cdot E_{p_{l}}^{\mu_{l}}\left(\frac{z}{z_{l}}\right) \cdot\left(z-z_{i}\right)^{j} \cdot F_{0}(z)} \cdot f(z) d z \\
& =\frac{2 \pi i}{(j-1) !} \frac{d^{j-1}}{d z^{j-1}}\left(\prod_{n=N+1}^{\infty} E_{p_{n}}^{m_{n}}\left(\frac{z}{z_{n}}\right) \cdot a(z) \cdot f(z)\right)\left(z_{i}\right) .
\end{aligned}
$$

This is precisely the equation which we obtained in (42). Therefore the same choice of $f$ and the sequences $p_{n}^{N}$ will lead to a contradiction. This completes the proof. Note that in each case $\left\langle T_{F_{N}} f, \varphi\right\rangle=0$. So this term does not contribute to estimate (46). 
We can now complete the proof of Theorem 1.

Proof of Theorem 1. The proof is standard and elementary (Note however that we use such a powerful tool as the closed graph theorem). We include it for completeness.

Assume that $T_{F}$ is an injective Fredholm operator. We shall show that $T_{F}$ is left invertible. If $T_{F}$ is additionally surjective then it is invertible. Thus assume that the classes of the functions $g_{1}, \ldots, g_{n} \in \mathcal{A}(\mathbb{R})$ in $\mathcal{A}(\mathbb{R}) / R\left(T_{F}\right)$ form a basis of $\mathcal{A}(\mathbb{R}) / R\left(T_{F}\right)$. For any $f \in \mathcal{A}(\mathbb{R})$ there are unique numbers $\alpha_{1}, \ldots, \alpha_{n}$ and a unique function $g \in \mathcal{A}(\mathbb{R})$ such that

$$
f=\alpha_{1} g_{1}+\cdots+\alpha_{n} g_{n}+T_{F} g .
$$

The uniqueness follows from the fact that $T_{F}$ is injective by assumption. We now define a left inverse operator of $T_{F}$. If $f$ is represented by (47) we put $S f=g$. The definition is correct, since the representation (47) is unique. By the same reason $S$ is linear. It is clear that

$$
S T_{F}=I \text {. }
$$

We show that $S$ is continuous. We apply the closed graph theorem ([36], Theorem 24.31). Observe that the space of real analytic functions is ultrabornological, i.e. it has the inductive topology of a family of Banach spaces and has a web (see [36], p. 287). The second statement follows from the fact that $\mathcal{A}(\mathbb{R})$ carries the projective topology of a sequence of ( $D F S$ )-spaces (see [36], Lemma 24.28, Corollary 24.29). Assume that $f_{\nu} \rightarrow 0$ and $S f_{\nu} \rightarrow h$ in $\mathcal{A}(\mathbb{R})$. We need to show that $h \equiv 0$. We have

$$
f_{\nu}=\alpha_{1}^{\nu} g_{1}+\cdots \alpha_{n}^{\nu} g_{n}+T_{F} g_{\nu} .
$$

It follows that the classes of $f_{\nu}$ in $\mathcal{A}(\mathbb{R}) / R\left(T_{F}\right)$ tend to zero in the quotient space. Hence, since the space $\mathcal{A}(\mathbb{R}) / R\left(T_{F}\right)$ is finite dimensional we have $\alpha_{i}^{\nu} \rightarrow$ 0 for $1 \leq i \leq n$. Also, $S f_{\nu}=g_{\nu} \rightarrow h$ by assumption. Hence, since $T_{F}$ is continuous

$$
f_{\nu} \rightarrow T_{F} h,
$$

and also $f_{\nu} \rightarrow 0$. Hence $T_{F} h=0$, which implies that $h=0$, since $T_{F}$ is injective. We infer that $S$ is a continuous linear left inverse of $T_{F}$.

If $T_{F}$ is left invertible, then the range $R\left(T_{F}\right)$ is a closed complemented subspace of $\mathcal{A}(\mathbb{R})$. It follows from Theorems 2.2 and 3 that $F=[\tilde{F}]$ in $\mathcal{X}(\mathbb{R})=$ limind $H(U \backslash K)$ for some $\tilde{F} \in H(U \backslash K)$ which does not vanish in $U \backslash K$. It follows from Theorem 2.1 that $T_{F}$ is a Fredholm operator. As a left invertible operator it must be injective.

It is a consequence of Theorem 3.1 that $T_{F}$ can only be injective if winding $\tilde{F} \geq 0$.

It follows from the Wiener-Hopf factorization (Theorem 3.2 above) that we can also give a constructive proof of left invertibility of injective Fredholm Toeplitz operators. First of all observe that

$$
T_{F G}=\mathcal{C} M_{F G}=\mathcal{C} M_{F}(\mathcal{C}+\mathcal{Q}) M_{G}=T_{F} T_{G}+\mathcal{C} M_{F} \mathcal{Q} M_{G},
$$


where $\mathcal{Q}$ denotes the complementary projection, i.e. $\mathcal{Q}:=I-\mathcal{C}$. It follows that if $G \in \mathcal{A}(\mathbb{R})$ or $F \in H_{0}\left(\mathbb{C}_{\infty} \backslash \mathbb{R}\right)$ then $\mathcal{C} M_{F} \mathcal{Q} M_{G}=0$ on $\mathcal{A}(\mathbb{R})$. As a result, if $F \in H_{0}\left(\mathbb{C}_{\infty} \backslash \mathbb{R}\right)$ or $G \in \mathcal{A}(\mathbb{R})$, then

$$
T_{F G}=T_{F} T_{G} \text {. }
$$

Since any $F \in H\left(\mathbb{C}_{\infty} \backslash \mathbb{R}\right)$ can be written as $F(\infty)+F_{0}$ with $F_{0} \in H_{0}\left(\mathbb{C}_{\infty} \backslash \mathbb{R}\right)$ we also have $(48)$ when $F \in H\left(\mathbb{C}_{\infty} \backslash \mathbb{R}\right)$ or $G \in \mathcal{A}(\mathbb{R})$.

Assume now that $T_{F}$ is a Fredholm operator and $k:=\operatorname{index} T_{F} \leq 0$. That is, $T_{F}$ is an injective Fredholm operator. Then it follows from Theorem 3.2 that $F$ can be factored as

$$
F=F_{-} z^{-k} F_{+}
$$

with $F_{-} \in H\left(\mathbb{C}_{\infty} \backslash \mathbb{R}\right)$ and $F_{+} \in \mathcal{A}(\mathbb{R})$ and both symbols are invertible in the corresponding spaces. Now we have

$$
T_{z^{k} F_{+}^{-1}} T_{F_{-}^{-1}} T_{F}=T_{z^{k} F_{+}^{-1}} T_{F_{-}^{-1}} T_{F_{-}} T_{z^{-k} F_{+}}=T_{z^{k} F_{+}^{-1}} T_{z^{-k} F_{+}}=I,
$$

since $F_{-}, F_{-}^{-1} \in H\left(\mathbb{C}_{\infty} \backslash \mathbb{R}\right)$ and $z^{-k} F_{+} \in \mathcal{A}(\mathbb{R})$. In other words, if $T_{F}$ is an injective Fredholm operator, then $T_{z^{k} F_{+}^{-1}} T_{F_{-}^{-1}}$ is a left inverse. One notices that this part of the theory is indeed very similar to the classical Hardy space case.

\subsection{Right Invertibility of Toeplitz Operators}

Our goal now is to prove Theorem 4 . Assume that $T_{F}: \mathcal{A}(\mathbb{R}) \rightarrow \mathcal{A}(\mathbb{R})$ is right invertible,

$$
T_{F} S=I
$$

Then $T_{F}^{\prime}: \mathcal{A}(\mathbb{R})_{b}^{\prime} \rightarrow \mathcal{A}(\mathbb{R})_{b}^{\prime}$ is left invertible. Hence, its range in $\mathcal{A}(\mathbb{R})_{b}^{\prime}$ is complemented. Also, if $T_{F}$ is right invertible then it is surjective. We show that if $F$ has non-real zeros accumulating at a real point but no real zeros going to infinity then the range of $T_{F}^{\prime}$ is not complemented in $\mathcal{A}(\mathbb{R})_{b}^{\prime}$. As a result, it follows from Theorem 4 that $T_{F}$ can be right invertible only if $T_{F}$ is a surjective Fredholm operator.

We start with the following easy observation.

Proposition 4.10. Assume that $F \in \mathcal{X}(\mathbb{R})$ is the equivalence class of $\tilde{F} \in$ $H(U \backslash K), U$ an open neighborhood of $\mathbb{R}, K$ a compact subset of $\mathbb{R}$ and $\tilde{F}$ vanishes only on a sequence $z_{n} \in U \backslash \mathbb{R}$, which accumulates at points of $K$ only. Then the range of $T_{F}^{\prime}$ is a closed subspace of $H_{0}\left(\mathbb{C}_{\infty} \backslash \mathbb{R}\right)$.

In view of Theorem 2.2 this Proposition will be proved once we have shown the following Lemma.

Lemma 4.11. If an operator $T: \mathcal{A}(\mathbb{R}) \rightarrow \mathcal{A}(\mathbb{R})$ is surjective then the image of the adjoint operator $T^{\prime}: \mathcal{A}(\mathbb{R})_{b}^{\prime} \rightarrow \mathcal{A}(\mathbb{R})_{b}^{\prime}$ is closed.

Proof. Since $\operatorname{ker} T=\left(R\left(T^{\prime}\right)\right)^{\circ}$ we have $(\operatorname{ker} T)^{\circ}=\left(R\left(T^{\prime}\right)\right)^{\circ \circ} \supset R\left(T^{\prime}\right)$. We show that the equality holds, which completes the proof since $(\operatorname{ker} T)^{\circ}$ is closed in the strong topology of $\mathcal{A}(\mathbb{R})^{\prime}$. To show that $(\operatorname{ker} T)^{\circ} \subset R\left(T^{\prime}\right)$ we define the map $T_{0}: \mathcal{A}(\mathbb{R}) / \operatorname{ker} T \rightarrow \mathcal{A}(\mathbb{R}), T_{0}(x+\operatorname{ker} T):=T x$. The map $T_{0}$ 
is a continuous linear bijection. The space $\mathcal{A}(\mathbb{R})$ is ultrabornological. Since $\mathcal{A}(\mathbb{R})$ has a web, so does the quotient $\mathcal{A}(\mathbb{R}) / \operatorname{ker} T$ ([36], Lemma 24.28). Hence the inverse map $T_{0}^{-1}: \mathcal{A}(\mathbb{R}) \rightarrow \mathcal{A}(\mathbb{R}) / \operatorname{ker} T$ is continuous. Take $\xi \in(\operatorname{ker} T)^{\circ}$ and define $\bar{\xi}:(x+\operatorname{ker} T) \mapsto \xi(x), \bar{\xi} \in(\mathcal{A}(\mathbb{R}) / \operatorname{ker} T)^{\prime}$. Let $\eta:=\bar{\xi} \circ T_{0}^{-1}$. Then $\eta \in \mathcal{A}(\mathbb{R})^{\prime}$. For $x \in \mathcal{A}(\mathbb{R})$ we have

$$
\left\langle T^{\prime} \eta, x\right\rangle=\langle\eta, T x\rangle=\left\langle\eta, T_{0}(x+\operatorname{ker} T)\right\rangle=\bar{\xi}(x+\operatorname{ker} T)=\xi(x) .
$$

That is $T^{\prime} \eta=\xi$. Since $\xi \in(\operatorname{ker} T)^{\circ}$ was arbitrary we have $(\operatorname{ker} T)^{\circ}=R\left(T^{\prime}\right)$.

Lemma 4.12. If $f, g \in H_{0}\left(\mathbb{C}_{\infty} \backslash K\right)$ and $\gamma$ is a $C^{\infty}$ smooth Jordan curve with $K \subset I(\gamma)$, then

$$
\int_{\gamma} f g d z=0
$$

Proof. We have

$$
\int_{\gamma} f g d z=\int_{|z|=R}\left(\sum_{n=1}^{\infty} \frac{f_{-n}}{z^{n}}\right)\left(\sum_{n=1}^{\infty} \frac{g_{-n}}{z^{n}}\right) d z=0,
$$

for $R>0$ large enough, since $\int_{|z|=R} z^{n}=0$ if $n \neq-1$.

Lemma 4.13. Assume that $G \in H(U \backslash K), U$ an open simply connected neighborhood of $\mathbb{R}, K$ a compact subset of $\mathbb{R}$. If for every $k \in \mathbb{N}$,

$$
\int_{\gamma} G(z) z^{-k} d z=0
$$

where $\gamma$ is a $C^{\infty}$ smooth Jordan curve in $U \backslash K$ with $K \subset I(\gamma)$, then $G \in$ $H_{0}\left(\mathbb{C}_{\infty} \backslash K\right)$.

Proof. By Cauchy's integral formula $G=G_{+}+G_{-}$, where $G_{+} \in H(U)$ and $G_{-} \in H_{0}\left(\mathbb{C}_{\infty} \backslash K\right)$. We have

$$
\begin{aligned}
0=\int_{\gamma} G(z) z^{-k} d z & =\int_{\gamma}\left(G_{+}(z)+G_{-}(z)\right) z^{-k} d z=\int_{\gamma} G_{+}(z) z^{-k} d z \\
& =\int_{|z|=\varepsilon}\left(\sum_{n=0}^{\infty} G_{n} z^{n}\right) z^{-k} d z,
\end{aligned}
$$

for sufficiently small $\varepsilon>0$. This implies that $G_{n}=0$ for $n \in \mathbb{N}$. The fact that $\int_{\gamma} G_{-}(z) z^{-k} d z=0$ follows from Lemma 4.12.

Let us recall that $\tilde{F} \in H(U \backslash K)$ vanishes only at $z_{n} \in U \backslash \mathbb{R}$ and the sequence $z_{n}$ accumulates only at points of $K$. As before we simplify the notation and write $F$ instead of $\tilde{F}$. For any $z_{n}$ let $w_{n} \in K$ be chosen in such a way that

$$
\left|z_{n}-w_{n}\right|=\operatorname{dist}\left(z_{n}, K\right) .
$$

We assume that

$$
\operatorname{dist}\left(z_{1}, K\right) \geq \operatorname{dist}\left(z_{2}, K\right) \geq \cdots
$$


Naturally, there exists only a finite number of points $z_{n}$ with the same distance to the set $K$. We factorize out the zeros of $F$,

$$
F(z)=\prod_{n=1}^{\infty} E_{p_{n}}^{m_{n}}\left(\frac{z_{n}-w_{n}}{z-w_{n}}\right) \cdot F_{0}(z),
$$

where $F_{0}$ does not vanish in $U \backslash K$ and $m_{n}$ is the multiplicity of $z_{n}$. Again at this moment the numbers $p_{n}$ are chosen only to guarantee that the product converges on compact subsets of $\mathbb{C} \backslash K$.

Proposition 4.14. The operator $T_{F_{0}}^{\prime}: H_{0}\left(\mathbb{C}_{\infty} \backslash \mathbb{R}\right) \rightarrow H_{0}\left(\mathbb{C}_{\infty} \backslash \mathbb{R}\right)$ is a Fredholm operator and

$$
\operatorname{index} T_{F_{0}}^{\prime}=-\operatorname{index} T_{F_{0}}=\text { winding } F_{0} \text {. }
$$

Proof. It follows from the Coburn-Simonenko theorem (Theorem 3.1) and the fact that the range of $T_{F_{0}}$ is closed ([9], Proposition 5.1) that $T_{F_{0}}$ is either injective or surjective. A finite dimensional subspace of a locally convex space is complemented. This follows from the fact that such a space is isomorphic with $\mathbb{C}^{n}$ and the Hahn-Banach Theorem. Hence the kernel of $T_{F_{0}}$ is a complemented subspace of $\mathcal{A}(\mathbb{R})$. In the proof of Theorem 1 we showed that if the range of $T_{F_{0}}$ is of positive codimension, then it is complemented. Furthermore, according to ([26], Theorem 13.5.2) a subspace of finite codimension is bornological. Since the range of $T_{F_{0}}$ is closed in the complete space $\mathcal{A}(\mathbb{R})$, it is ultrabornological ([36], Remark 24.15 (b)). The open mapping theorem ([36], Theorem 24.30) implies that $T_{F_{0}}$ maps open sets in $\mathcal{A}(\mathbb{R})$ into open sets in $R\left(T_{F_{0}}\right)$. According to ([39], Satz 1.1) the operator $T_{F_{0}}$ is a $\varrho$-transformation ([39], Definition 1.1). According to ([39], Satz 7.1) the adjoint of $T_{F_{0}}$ on $\mathcal{A}(\mathbb{R})_{b}^{\prime}$ is a $\varrho$-transformation. Furthermore,

$$
\operatorname{dim} \operatorname{ker} T_{F_{0}}=\operatorname{codim} R\left(T_{F_{0}}^{\prime}\right), \quad \operatorname{codim} R\left(T_{F_{0}}\right)=\operatorname{dim} \operatorname{ker} T_{F_{0}} .
$$

Let us recall that the space $\mathcal{A}(\mathbb{R})$ is reflexive. This implies that any continuous linear functional on $\mathcal{A}(\mathbb{R})_{b}^{\prime}$ is determined by some $f \in \mathcal{A}(\mathbb{R})$ in the sense of duality (15). To denote the kernel of this functional we simply write $\operatorname{ker} f$.

Lemma 4.15. Assume that $F_{0} \in H(U \backslash K), U$ an open simply connected neighborhood of $\mathbb{R}, K$ a compact subset of $\mathbb{R}$, does not vanish in $U \backslash K$. Furthermore, assume that index $T_{F_{0}}^{\prime}=$ winding $F_{0}=-1$. Then there exists a function $f \in \mathcal{A}(\mathbb{R})$ such that

$$
R\left(T_{F_{0}}^{\prime}\right)=\operatorname{ker} f
$$

Proof. The range of $T_{F_{0}}^{\prime}$ is a closed subspace of $H_{0}\left(\mathbb{C}_{\infty} \backslash \mathbb{R}\right)$. This follows from the arguments of ([9], Proposition 5.1), since one can use the open mapping theorem ([36], Theorem 24.30) for the Fredholm operator $T_{F_{0}}^{\prime}$. Indeed, $\mathcal{A}(\mathbb{R})$ is a complete nuclear space. Hence it is a Schwartz space and, as a result, $\mathcal{A}(\mathbb{R})_{b}^{\prime}$ is ultrabornological ([36], Proposition 24.23). Also, by ([36], Lemma $24.28)$ the space $H_{0}\left(\mathbb{C}_{\infty} \backslash \mathbb{R}\right)$ is webbed. 
Notice that if index $T_{F_{0}}^{\prime}=-1$, then $\operatorname{dim} \operatorname{ker} T_{F_{0}}^{\prime}=0$ and, as a result $\operatorname{dim}$ coker $T_{F_{0}}^{\prime}=1$. Indeed, by Proposition 4.14 we have index $T_{F_{0}}=1$, which implies that $T_{F_{0}}$ is not injective. In view of the Coburn-Simonenko Theorem $T_{F_{0}}^{\prime}$ is injective (Theorem 3.1). Since $\mathcal{A}(\mathbb{R})$ is reflexive, it suffices to apply the arguments from Lemma 4.5.

Lemma 4.16. Assume that $F_{0} \in H(U \backslash K)$ does not vanish, $U$ an open simply connected neighborhood of $\mathbb{R}, K$ a compact subset of $\mathbb{R}$. Furthermore, assume that

$$
\operatorname{index} T_{F_{0}}^{\prime}=\text { winding } F_{0}=-1 .
$$

Let $f \in \mathcal{A}(\mathbb{R})$ be as in the previous Lemma, i.e.

$$
R\left(T_{F_{0}}^{\prime}\right)=\operatorname{ker} f .
$$

For $N \in \mathbb{N}$ consider the symbol function

$$
G(z)=\prod_{n=1}^{N-1} E_{p_{n}}^{m_{n}}\left(\frac{z_{n}-w_{n}}{z-w_{n}}\right) \cdot E_{p_{N}}^{l}\left(\frac{z_{n}-w_{n}}{z-w_{n}}\right) \cdot F_{0}(z):=\mathcal{E}(z) \cdot F_{0}(z)
$$

with $l \leq m_{N}$. Then

$$
R\left(T_{G}^{\prime}\right)=\operatorname{ker} f \cap \bigcap_{n=1}^{N-1} \bigcap_{k=1}^{m_{n}} \operatorname{ker} \frac{f}{\left(z-z_{n}\right)^{k}} \cap \operatorname{ker} \frac{f}{\left(z-z_{N}\right)} \cap \cdots \cap \operatorname{ker} \frac{f}{\left(z-z_{N}\right)^{l}} .
$$

In other words, if $g$ vanishes on $R\left(T_{G}^{\prime}\right)$, then

$$
\begin{aligned}
g \in & \operatorname{Span}\left\{f, \frac{f}{\left(z-z_{1}\right)}, \ldots, \frac{f}{\left(z-z_{1}\right)^{m_{1}}}, \ldots, \frac{f}{\left(z-z_{N-1}\right)}, \ldots,\right. \\
& \left.\frac{f}{\left(z-z_{N-1}\right)^{m_{N-1}}}, \frac{f}{\left(z-z_{N}\right)}, \ldots, \frac{f}{\left(z-z_{N}\right)}\right\} .
\end{aligned}
$$

Observe that since the zeros $\left(z_{n}\right)$ are not real, the function $\frac{f}{\left(z-z_{n}\right)^{k}}$ belongs to $\mathcal{A}(\mathbb{R})$.

Proof. Observe that it follows from Proposition 4.14 that

$$
\text { index } \begin{aligned}
T_{G}^{\prime} & =-\operatorname{index} T_{G}=\operatorname{winding} G \\
& =-\left(m_{1}+\cdots+m_{N-1}+l\right)+\text { winding } F_{0} \\
& =-\left(m_{1}+\cdots+m_{N-1}+l\right)-1 .
\end{aligned}
$$

This implies that $T_{G}$ is not injective and, as a result, by the Coburn Simonenko Theorem, $\operatorname{ker} T_{G}^{\prime}=\{0\}$. Hence $\operatorname{dim} \operatorname{coker} T_{G}^{\prime}=m_{1}+\cdots+m_{N-1}+$ $l+1$. The rest of the proof follows from arguments similar to the proof of Lemma 4.6. Consider the family

$$
\begin{aligned}
\mathcal{G}:= & \left\{f, \frac{f}{\left(z-z_{1}\right)}, \ldots, \frac{f}{\left(z-z_{1}\right)^{m_{1}}}, \ldots, \frac{f}{\left(z-z_{N-1}\right)}, \ldots, \frac{f}{\left(z-z_{N-1}\right)^{m_{N-1}}},\right. \\
& \left.\frac{f}{\left(z-z_{N}\right)}, \ldots, \frac{f}{\left(z-z_{N}\right)^{l}}\right\} .
\end{aligned}
$$


We have $R\left(T_{G}^{\prime}\right) \subset \bigcap_{g \in \mathcal{G}}$ ker $g$. Indeed, for $g \in \mathcal{G}$ we have

$$
\begin{aligned}
\left\langle g, T_{G}^{\prime} \phi\right\rangle & =\left\langle T_{G} g, \phi\right\rangle=\int_{\gamma} G(z) g(z) \phi(z) d z=\int_{\gamma} \frac{\mathcal{E}(z)}{\left(z-z_{n}\right)^{j}} F_{0}(z) \phi(z) f(z) d z \\
& =\left\langle f, T_{F_{0}}^{\prime} \psi\right\rangle
\end{aligned}
$$

with $\psi=\frac{\mathcal{E}}{\left(z-z_{n}\right)^{j}} \cdot \phi \in H_{0}\left(\mathbb{C}_{\infty} \backslash \mathbb{R}\right)$. Since $f$ vanishes on the range of $T_{F_{0}}^{\prime}$ we have $\left\langle g, T_{G}^{\prime} \phi\right\rangle=0$. Since the functions in $\mathcal{G}$ are linearly independent, the proof is complete.

Lemma 4.16 is now used to obtain an analog of Proposition 4.3.

Proposition 4.17. Let $F \in H(U \backslash K)$ vanish on $z_{n} \in U \backslash \mathbb{R}$ only. The sequence $\left(z_{n}\right)$ accumulates only at points of $K$. If index $T_{F_{0}}^{\prime}=-1$, then there exists a function $f \in H(V), V$ an open neighborhood of $\mathbb{R}$, such that

$$
R\left(T_{F}^{\prime}\right)=\operatorname{ker} f \cap \bigcap_{n=1}^{\infty} \bigcap_{j=1}^{m_{n}} \operatorname{ker} \frac{f}{\left(z-z_{n}\right)^{j}} .
$$

If index $T_{F_{0}}^{\prime}=k \geq 0$, then there exists a function $f \in H(V)$ and natural numbers $l, \mu_{l}, \mu_{l} \leq m_{l}$ such that

$$
R\left(T_{F}^{\prime}\right)=\operatorname{ker} f \cap \bigcap_{j=1}^{m_{l}-\mu_{l}} \operatorname{ker} \frac{f}{\left(z-z_{l}\right)^{j}} \cap \bigcap_{n=l+1}^{\infty} \bigcap_{j=1}^{m_{n}} \operatorname{ker} \frac{f}{\left(z-z_{n}\right)^{j}} .
$$

If index $T_{F_{0}}^{\prime}=k<-1$, then there exists a function $f \in H(V)$ and natural numbers $l, \mu_{l}$,

$$
\begin{aligned}
& R\left(T_{F}^{\prime}\right) \\
& =\operatorname{ker} f \cap \bigcap_{j=1}^{m_{l}+\mu_{l}} \operatorname{ker} \frac{f}{\left(z-z_{l}\right)^{j}} \cap \bigcap_{i=1}^{l-1} \bigcap_{j=1}^{2 m_{i}} \operatorname{ker} \frac{f}{\left(z-z_{i}\right)^{j}} \cap \bigcap_{i=l+1}^{\infty} \bigcap_{j=1}^{m_{i}} \operatorname{ker} \frac{f}{\left(z-z_{i}\right)^{j}} .
\end{aligned}
$$

Also, if index $T_{F_{0}}^{\prime}=k \leq-1$, then there exists a function $a \in H_{0}\left(\mathbb{C}_{\infty} \backslash K\right)$ such that

$$
f(z)=\frac{a(z)}{F_{0}(z)}
$$

for $z \in(U \cap V) \backslash K$. If index $T_{F_{0}}^{\prime} \geq 0$, then there exists a function $a \in$ $H_{0}\left(\mathbb{C}_{\infty} \backslash K\right)$ such that

$$
f(z)=\frac{a(z)}{\prod_{n=1}^{l-1} E_{p_{n}}^{m_{n}}\left(\frac{z_{n}-w_{n}}{z-w_{n}}\right) \cdot E_{p_{l}}^{\mu_{l}}\left(\frac{z_{n}-w_{n}}{z-w_{n}}\right) \cdot F_{0}(z)}
$$

for $z \in(U \cap V) \backslash K$ and $z \neq z_{1}, \ldots, z_{l}$.

Proof. We essentially follow the arguments from the proof of Proposition 4.3. Assume first that index $T_{F_{0}}^{\prime}=-1$. As in (49) we show that

$$
R\left(T_{F}^{\prime}\right) \subset \operatorname{ker} f \cap \bigcap_{n=1}^{\infty} \bigcap_{j=1}^{m_{n}} \operatorname{ker} \frac{f}{\left(z-z_{n}\right)^{j}}
$$


To show the equality assume that $g \in H(V)$ vanishes on the range of $T_{F}^{\prime}$, i.e. $\left\langle g, T_{F}^{\prime} \phi\right\rangle=0$ for any $\phi \in H_{0}\left(\mathbb{C}_{\infty} \backslash \mathbb{R}\right)$. It follows from Lemma 4.13 that there exists a function $a \in H_{0}\left(\mathbb{C}_{\infty} \backslash K\right)$ such that

$$
a(z)=F(z) \cdot g(z)=\prod_{n=1}^{\infty} E_{p_{n}}^{m_{n}}\left(\frac{z_{n}-w_{n}}{z-w_{n}}\right) \cdot F_{0}(z) \cdot g(z)
$$

for $z \in(U \cap V) \backslash K$. There is $N \in \mathbb{N}$ such that $z_{N+1}, \ldots \in U \cap V$. Thus for a different function $b \in H_{0}\left(\mathbb{C}_{\infty} \backslash K\right)$ it holds that

$$
b(z)=\prod_{n=1}^{N} E_{p_{n}}^{m_{n}}\left(\frac{z_{n}-w_{n}}{z-w_{n}}\right) \cdot F_{0}(z) \cdot g(z)
$$

for $z \in(U \cap V) \backslash K$. Recall that

$$
\lim _{z \rightarrow \infty} \prod_{n=N+1}^{\infty} E_{p_{n}}^{m_{n}}\left(\frac{z_{n}-w_{n}}{z-w_{n}}\right)=1 .
$$

For $z \in(U \cap V) \backslash K$ we have

$$
g(z)=\frac{1}{\prod_{n=1}^{N} E_{p_{n}}^{m_{n}}\left(\frac{z_{n}-w_{n}}{z-w_{n}}\right)} \cdot \frac{b(z)}{F_{0}(z)} .
$$

Put now

$$
G_{N}(z)=\prod_{n=1}^{N} E_{p_{n}}^{m_{n}}\left(\frac{z_{n}-w_{n}}{z-w_{n}}\right) \cdot F_{0}(z) .
$$

It follows from Lemma 4.12 that $\left\langle g, T_{G_{N}}^{\prime} \phi\right\rangle=0$ for any $\phi \in H_{0}\left(\mathbb{C}_{\infty} \backslash \mathbb{R}\right)$. We apply now Lemma 4.16 to conclude that

$$
\begin{aligned}
g \in & \operatorname{Span}\left\{f, \frac{f}{\left(z-z_{1}\right)}, \ldots, \frac{f}{\left(z-z_{1}\right)^{m_{1}}}, \ldots,\right. \\
& \left.\frac{f}{\left(z-z_{N-1}\right)}, \ldots, \frac{f}{\left(z-z_{N-1}\right)^{m_{N-1}}}, \frac{f}{\left(z-z_{N}\right)}, \ldots, \frac{f}{\left(z-z_{N}\right)^{m_{N}}}\right\} .
\end{aligned}
$$

It follows now from the Hahn-Banach theorem that

$$
R\left(T_{F}^{\prime}\right)=\operatorname{ker} f \cap \bigcap_{n=1}^{\infty} \bigcap_{j=1}^{m_{n}} \operatorname{ker} \frac{f}{\left(z-z_{n}\right)^{j}} .
$$

Consider now the case index $T_{F_{0}}^{\prime}=k \geq 0$. Choose $l$ and $\mu_{l}$ such that

$$
m_{0}+m_{1}+\cdots+m_{l-1}+\mu_{l}=k+1 \text {. }
$$

If

$$
G(z)=\prod_{n=1}^{l-1} E_{p_{n}}^{m_{n}}\left(\frac{z_{n}-w_{n}}{z-w_{n}}\right) \cdot E_{p_{l}}^{\mu_{l}}\left(\frac{z_{n}-w_{n}}{z-w_{n}}\right) \cdot F_{0}(z)
$$


then index $T_{G}^{\prime}=-1$. As as result, we can proceed as above and as in Lemma 4.7. If index $T_{F_{0}}^{\prime}=k<0$, then we put

$$
G(z)=\frac{F_{0}(z)}{\prod_{n=1}^{l-1} E_{p_{n}}^{m_{n}}\left(\frac{z_{n}-w_{n}}{z-w_{n}}\right) E_{p_{l}}^{\mu_{l}}\left(\frac{z_{n}-w_{n}}{z-w_{n}}\right)},
$$

where $m_{0}+m_{1}+\cdots+m_{l-1}+\mu_{l}=|k|-1$. Then index $T_{G}^{\prime}=-1$.

Assume now that index $T_{F_{0}}^{\prime}=-1$. Then $R\left(T_{F_{0}}^{\prime}\right)=\operatorname{ker} f$, which implies that

$$
\int_{\gamma} F_{0}(z) \varphi(z) f(z) d z=0
$$

for every $\varphi(z) \in H_{0}\left(\mathbb{C}_{\infty} \backslash \mathbb{R}\right)$. We have that $F_{0}(z) f(z)$ is holomorphic $(U \cap$ $V) \backslash K$. It follows from Lemma 4.13 that there exists $a \in H_{0}\left(\mathbb{C}_{\infty} \backslash K\right)$ such that

$$
F_{0}(z) f(z)=a(z)
$$

for $z \in(U \cap V) \backslash K$. That is,

$$
f(z)=\frac{a(z)}{F_{0}(z)}
$$

for $z \in(U \cap V) \backslash K$. If index $T_{F_{0}}^{\prime}<-1$ we have by (53),

$$
f(z) \cdot \frac{F_{0}(z)}{\prod_{n=1}^{l-1} E_{p_{n}}^{m_{n}}\left(\frac{z_{n}-w_{n}}{z-w_{n}}\right) E_{p_{l}}^{\mu_{l}}\left(\frac{z_{n}-w_{n}}{z-w_{n}}\right)}=a(z)
$$

for $a \in H_{0}\left(\mathbb{C}_{\infty} \backslash K\right)$. This gives the same representation (50) for a different function $a \in H_{0}\left(\mathbb{C}_{\infty} \backslash K\right)$, since $\lim _{z \rightarrow \infty} E_{p_{n}}\left(\frac{z_{n}-w_{n}}{z-w_{n}}\right)=1$. If index $T_{F_{0}}^{\prime} \geq 0$ we apply (52) in order to show (51).

We constructed functions $f_{j} \in \mathcal{A}(\mathbb{R})$ such that

$$
R\left(T_{F}^{\prime}\right)=\bigcap_{j=1}^{\infty} \operatorname{ker} f_{j}
$$

We now sketch the construction of functionals dual to $f_{j}$. To simplify the notation we assume that

$$
R\left(T_{F}^{\prime}\right)=\operatorname{ker} f \cap \bigcap_{n=1}^{\infty} \bigcap_{k=1}^{m_{n}} \operatorname{ker} \frac{f}{\left(z-z_{n}\right)^{k}} .
$$

Assume that index $T_{F_{0}}^{\prime} \leq-1$. Recall that

$$
f(z)=\frac{a(z)}{F_{0}(z)}
$$

for $z \in(U \cap V) \backslash K$, where $a \in H_{0}\left(\mathbb{C}_{\infty} \backslash K\right)$. Define

$$
A:=\left\{n \in \mathbb{N}: a\left(z_{n}\right)=0\right\} .
$$


It follows from (54) that $A$ is finite. For $n \in A$ let $\alpha_{n}$ be the multiplicity of $z_{n}$. Write

$$
a(z)=\prod_{i \in A}\left(z-z_{i}\right)^{\alpha_{i}} b(z)
$$

for $b \in H_{0}\left(\mathbb{C}_{\infty} \backslash K\right)$. Consider the symbol function

$$
\mathcal{F}_{n}(z):=\frac{\prod_{i \neq n} E_{p_{i}}^{m_{i}}\left(\frac{z_{i}-w_{i}}{z-w_{i}}\right)}{\prod_{i \in A}\left(z-z_{i}\right)^{\alpha_{i}}} \cdot F_{0}(z) .
$$

If $\varphi \in H_{0}\left(\mathbb{C}_{\infty} \backslash \mathbb{R}\right)$ is arbitrary and $l \neq n, \kappa \leq m_{n}$, then by $(54)$

$$
\left\langle\frac{f}{\left(z-z_{l}\right)^{\kappa}}, T_{\mathcal{F}_{n}}^{\prime} \varphi\right\rangle=\int_{\gamma} \frac{\prod_{i \neq n} E_{p_{i}}^{m_{i}}\left(\frac{z_{i}-w_{i}}{z-w_{i}}\right)}{\left(z-z_{l}\right)^{\kappa}} \varphi(z) b(z) d z=0,
$$

since $\lim _{z \rightarrow \infty} \prod_{i \neq n} E_{p_{i}}^{m_{i}}\left(\frac{z_{i}-w_{i}}{z-w_{i}}\right)=1$. We used Lemma 4.12. We need to choose functions $\varphi_{n, k} \in H_{0}\left(\mathbb{C}_{\infty} \backslash \mathbb{R}\right)$ such that

$$
\left\langle\frac{f}{\left(z-z_{n}\right)^{j}}, T_{\mathcal{F}_{n}}^{\prime} \varphi_{n, k}\right\rangle=\delta_{k j}, j=1, \ldots, m_{n}
$$

Assume that $\varphi_{n, k} \in H_{0}\left(\mathbb{C}_{\infty} \backslash[-N, N]\right)$ and $N$ is large enough to guarantee $K \subset[-N, N]$. We have

$$
\left\langle\frac{f}{\left(z-z_{n}\right)^{j}}, T_{\mathcal{F}_{n}}^{\prime} \varphi_{n, k}\right\rangle=\int_{\gamma} \frac{\prod_{i \neq n} E_{p_{i}}^{m_{i}}\left(\frac{z_{i}-w_{i}}{z-w_{i}}\right)}{\left(z-z_{n}\right)^{j}} \varphi_{n, k}(z) b(z) d z,
$$

where $\gamma$ is $C^{\infty}$ smooth Jordan curve in $(U \cap V) \backslash[-N, N]$ with $[-N, N] \subset I(\gamma)$ and $z_{n} \in E(\gamma)$. If $\Gamma$ is a $C^{\infty}$ smooth Jordan curve such that $\gamma \subset I(\Gamma)$ and $z_{n} \in I(\Gamma)$, it follows from the argument given in the proof of Lemma 4.12 that

$$
\int_{\Gamma} \frac{\prod_{i \neq n} E_{p_{i}}^{m_{i}}\left(\frac{z_{i}-w_{i}}{z-w_{i}}\right)}{\left(z-z_{n}\right)^{j}} \varphi_{n, k}(z) b(z) d z=0
$$

since the functions $b, \varphi_{n, k}$ vanish at infinity and $\lim _{z \rightarrow \infty} \prod_{i \neq n} E_{p_{i}}^{m_{i}}\left(\frac{z_{i}-w_{i}}{z-w_{i}}\right)=$ 1. Hence, by Cauchy's integral formula applied to the cycle $(-\gamma) \cup \Gamma$,

$$
\begin{aligned}
& \left\langle\frac{f}{\left(z-z_{n}\right)^{j}}, T_{\mathcal{F}_{n}}^{\prime} \varphi_{n, k}\right\rangle \\
& \quad=-\frac{2 \pi i}{(j-1) !} \frac{d^{j-1}}{d z^{j-1}}\left(\prod_{i \neq n} E_{p_{i}}^{m_{i}}\left(\frac{z_{i}-w_{i}}{z-w_{i}}\right) \varphi_{n, k}(z) b(z)\right)\left(z_{n}\right) .
\end{aligned}
$$

In order to find functions $\varphi_{n, k}$, which satisfy (55) one applies the transformation $z \mapsto \frac{1}{z}$ and works in $\mathbb{C} \backslash\left(\left(-\infty,-\frac{1}{N}\right) \cup\left(\frac{1}{N}, \infty\right)\right)$ imposing the additional condition that the function vanishes at 0 . This leads to a system of equations involving derivatives of the unknown functions at $z_{n}$, which can be solved inductively. 
Proof of Theorem 4. It suffices to show that if $F$ vanishes only on a sequence $\left(z_{n}\right) \subset \mathbb{C} \backslash \mathbb{R}$ which accumulates at points of $K$ only, then the range of the operator $T_{F}^{\prime}$ is not complemented. We mimic the proof of Theorem 3 .

Assume that there exists a continuous projection $P: H_{0}\left(\mathbb{C}_{\infty} \backslash \mathbb{R}\right) \rightarrow$ $H_{0}\left(\mathbb{C}_{\infty} \backslash \mathbb{R}\right)$ onto the range of the operator $T_{F}^{\prime}$. Denote

$$
X_{N}:=\left\{\varphi \in H_{0}\left(\mathbb{C}_{\infty} \backslash \mathbb{R}\right): f_{N+1}(\varphi)=f_{N+2}(\varphi)=\cdots=0\right\},
$$

where

$$
R\left(T_{F}^{\prime}\right)=\bigcap_{n=1}^{\infty} \operatorname{ker} f_{n}
$$

Denote also

$$
X=\bigcup_{N=1}^{\infty} X_{N}
$$

As in the proof of Theorem 3 we conclude that there exist functions $\psi_{n} \in$ $H_{0}\left(\mathbb{C}_{\infty} \backslash \mathbb{R}\right)$ such that for any $\varphi \in X$

$$
(I-P) \varphi=\sum_{n=1}^{\infty} f_{n}(\varphi) \psi_{n}
$$

None of the functions $\psi_{n}$ is identically equal to zero. There exists therefore $\tilde{z} \in \mathbb{C} \backslash \mathbb{R}$ such that $\psi_{n}(\tilde{z}) \neq 0$ for $n \in \mathbb{N}$. It suffices to construct a sequence $\phi_{n} \in X$ convergent in $H_{0}\left(\mathbb{C}_{\infty} \backslash \mathbb{R}\right)$ such that $\left((I-P) \phi_{n}\right)(\tilde{z})$ diverges, since the evaluation at $\tilde{z}$ is a continuous functional on $H_{0}\left(\mathbb{C}_{\infty} \backslash \mathbb{R}\right)$.

Let

$$
F_{N}(z):=\prod_{n=N+1}^{\infty} E_{p_{n}}^{m_{n}}\left(\frac{z_{n}-w_{n}}{z-w_{n}}\right) \cdot F_{0}(z)
$$

If $i>N$ and $1 \leq k \leq m_{i}$ then

$$
\left\langle\frac{f}{\left(z-z_{i}\right)^{k}}, T_{F_{N}}^{\prime} \varphi\right\rangle=\int_{\gamma} \frac{\prod_{n=N+1}^{\infty} E_{p_{n}}^{m_{n}}\left(\frac{z_{n}-w_{n}}{z-w_{n}}\right) \cdot F_{0}(z)}{\left(z-z_{i}\right)^{k}} \cdot \varphi(z) \cdot \frac{a(z)}{F_{0}(z)} d z=0
$$

by Lemma 4.12 , since $\lim _{z \rightarrow \infty} \prod_{n=N+1}^{\infty} E_{p_{n}}^{m_{n}}\left(\frac{z_{n}-w_{n}}{z-w_{n}}\right)=1$. Hence for every $\varphi \in H_{0}\left(\mathbb{C}_{\infty} \backslash \mathbb{R}\right)$, the function $T_{F_{N}}^{\prime} \varphi$ belongs to $X$. Consider now those points $z_{n}$, which satisfy $a\left(z_{n}\right) \neq 0$. Choose a function $\varphi \in H_{0}\left(\mathbb{C}_{\infty} \backslash \mathbb{R}\right)$ such that

$$
\frac{2 \pi i}{j !}(a \cdot \varphi)^{(j)}\left(z_{n}\right)=\frac{1}{\psi_{M}(\tilde{z})}, j=0, \ldots, m_{n}-1,
$$

where $\psi_{M}=(I-P) \varphi_{M}$ and $\varphi_{M}$ is dual to $f_{M}=\frac{f}{\left(z-z_{n}\right)^{j+1}}$. If $a\left(z_{n}\right)=0$ we take $\varphi$ to satisfy $\varphi\left(z_{n}\right)=\varphi^{\prime}\left(z_{n}\right)=\cdots=\varphi^{m_{n}-1}\left(z_{n}\right)=0$. Recall that $a\left(z_{n}\right)=0$ only for a finite number of $n \in \mathbb{N}$. We know that $T_{F_{N}}^{\prime} \varphi \in X$. It suffices therefore to choose numbers $p_{n}^{N}$ such that

$$
(I-P)\left(T_{F_{N}} \varphi\right)
$$


diverges at $\tilde{z}$, since

$$
\prod_{n=N+1}^{\infty} E_{p_{n}^{N}}^{m_{n}}\left(\frac{z_{n}-w_{n}}{z-w_{n}}\right) \cdot \varphi(z)
$$

will converge to $\varphi$ in $H_{0}\left(\mathbb{C}_{\infty} \backslash \mathbb{R}\right)$ as $N \rightarrow \infty$. Recall that

$$
\lim _{z \rightarrow \infty} \prod_{n=N+1}^{\infty} E_{p_{n}^{N}}^{m_{n}}\left(\frac{z_{n}-w_{n}}{z-w_{n}}\right)=1 .
$$

Hence,

$$
\prod_{n=N+1}^{\infty} E_{p_{n}^{N}}^{m_{n}}\left(\frac{z_{n}-w_{n}}{z-w_{n}}\right) \cdot \varphi(z) \in H_{0}\left(\mathbb{C}_{\infty} \backslash \mathbb{R}\right) .
$$

This implies that

$$
T_{F_{N}}^{\prime} \varphi=T_{F_{0}}^{\prime}\left(\prod_{n=N+1}^{\infty} E_{p_{n}^{N}}^{m_{n}}\left(\frac{z_{n}-w_{n}}{z-w_{n}}\right) \cdot \varphi(z)\right) \rightarrow T_{F_{0}}^{\prime} \varphi,
$$

since $T_{F_{0}}^{\prime}$ is continuous on $H_{0}\left(\mathbb{C}_{\infty} \backslash \mathbb{R}\right)$.

For $1 \leq i \leq N$ we have

$$
\begin{aligned}
\left\langle\frac{f}{\left(z-z_{i}\right)^{j}}, T_{F_{N}}^{\prime} \varphi\right\rangle & =\int_{\gamma} \frac{\prod_{n=N+1}^{\infty} E_{p_{n}^{N}}^{m_{n}}\left(\frac{z_{n}-w_{n}}{z-w_{n}}\right) \cdot \varphi(z) \cdot a(z)}{\left(z-z_{i}\right)^{j}} d z \\
& =-\frac{2 \pi i}{(j-1) !} \frac{d^{j-1}}{d z^{j-1}}\left(\prod_{n=N+1}^{\infty} E_{p_{n}^{N}}^{m_{n}}\left(\frac{z_{n}-w_{n}}{z-w_{n}}\right) \cdot \varphi(z) \cdot a(z)\right)\left(z_{i}\right)
\end{aligned}
$$

by Lemma 4.12 and Cauchy's integral formula. Without loss of generality we assume that

$$
\operatorname{dist}\left(z_{1}, K\right)>\operatorname{dist}\left(z_{2}, K\right)>\cdots .
$$

Otherwise we group the points $z_{n}$ into necessarily finite subsets of points of the same distance to $K$ and construct the sequences $p_{n}^{N}$ for these subsets. Choose numbers $\delta_{N}>0$ in such a way that $\operatorname{dist}\left(z_{N}, K\right)-\delta_{N}>\operatorname{dist}\left(z_{N+1}, K\right)$. We need to choose sequences $p_{n}^{N}$ in such a way that

$$
\left|\prod_{n=N+1}^{\infty} E_{p_{n}^{N}}^{m_{n}}\left(\frac{z_{n}-w_{n}}{z-w_{n}}\right)-1\right| \leq \frac{1}{3} \varepsilon_{N}
$$

for $z$ with $\operatorname{dist}(z, K) \geq \operatorname{dist}\left(z_{N}, K\right)-\delta_{N}$. We use Cauchy's integral formula as in the proof of Theorem 3 to determine the appropriate numbers $\varepsilon_{N}$. Observe that we may assume that $K$ is a closed interval and, as a result, the set of points $z$ with $\operatorname{dist}(z, K)=\operatorname{dist}\left(z_{N}, K\right)-\delta_{N}$ is a piecewise smooth curve.

For $z$ with $\operatorname{dist}(z, K) \geq \operatorname{dist}\left(z_{N}, K\right)-\delta_{N}$ and $n \geq N+1$ we have

$$
\begin{aligned}
\left|\frac{z_{n}-w_{n}}{z-w_{n}}\right| & \leq\left|z_{n}-w_{n}\right|[\operatorname{dist}(z, K)]^{-1} \leq\left|z_{n}-w_{n}\right|\left[\operatorname{dist}\left(z_{N}, K\right)-\delta_{N}\right]^{-1} \\
& =\frac{\operatorname{dist}\left(z_{n}, K\right)}{\operatorname{dist}\left(z_{N}, K\right)-\delta_{N}}<1
\end{aligned}
$$


by the choice of the numbers $\delta_{N}$. Also, for $z$ with $\operatorname{dist}(z, K) \geq \operatorname{dist}\left(z_{N}, K\right)-$ $\delta_{N}$ it holds that

$$
\begin{aligned}
\left|\sum_{n=N+1}^{\infty} \log E_{p_{n}^{N}}^{m_{n}}\left(\frac{z_{n}-w_{n}}{z-w_{n}}\right)\right| & \leq \frac{3}{2} \sum_{n=N+1} m_{n}\left|E_{p_{n}^{N}}\left(\frac{z_{n}-w_{n}}{z-w_{n}}\right)-1\right| \\
& \leq \frac{3}{2} \sum_{n=N+1}^{\infty} m_{n}\left(\frac{\operatorname{dist}\left(z_{n}, K\right)}{\operatorname{dist}\left(z_{N}, K\right)-\delta_{N}}\right)^{p_{n}^{N}+1} .
\end{aligned}
$$

In order to complete the proof it suffices to choose the numbers $p_{n}^{N}$ is such a way that

$$
\sum_{n=N+1}^{\infty} m_{n}\left(\frac{\operatorname{dist}\left(z_{n}, K\right)}{\operatorname{dist}\left(z_{n-1}, K\right)-\delta_{n-1}}\right)^{p_{n}^{N}+1}<\epsilon_{N}
$$

for the number $\epsilon_{N}$ such that if $|z|<\eta_{N}$ then $\left|e^{z}-1\right|<\eta_{N} / 3$. An estimate similar to (46) completes the proof.

We now complete the proof of Theorem 2.

Proof of Theorem 2. We show that a surjective Fredholm Toeplitz operator $T_{F}$ is right invertible. Since $T_{F}$ is a Fredholm operator, $\operatorname{ker} T_{F}$ is finite dimensional. A finite dimensional subspace of a locally convex space is complemented. Indeed, such a finite dimensional subspace $E$ is isomorphic with $\mathbb{C}^{n}$. Hence there exists a basis $e_{1}, \ldots, e_{n}$ in $E$ with coefficient functionals $f_{1}, \ldots, f_{n}$. By the Hahn-Banach theorem the functionals $f_{j}$ extend to continuous functionals on the whole space. This suffices to construct a continuous projection onto $E$.

Let $P: \mathcal{A}(\mathbb{R}) \rightarrow \mathcal{A}(\mathbb{R})$ be a continuous projection onto $\operatorname{ker} T_{F}$. The operator $T_{F}$ when restricted to the closed space $Y:=(I-P)(\mathcal{A}(\mathbb{R}))$ is injective. For any $g \in \mathcal{A}(\mathbb{R})$ there exists exactly one $f \in Y$ such that $g=T_{F} f$. We set $S g=f$. Since $\left.T_{F}\right|_{Y}$ is injective, the map $S$ is linear. It is obviously a right inverse of $T_{F}$. In order to show that $S$ is continuous we apply the closed graph theorem ([36], Theorem 24.31). This is legitimate since the space $\mathcal{A}(\mathbb{R})$ is both webbed and ultrabornological. Assume that $g_{\alpha}=T_{F} f_{\alpha}$ with $f_{\alpha} \in Y$ converges to 0 and also $f_{\alpha} \rightarrow f$. Since $Y$ is closed, $f \in Y$. Furthermore, since $T_{F}$ is continuous $T_{F} f_{\alpha} \rightarrow T_{F} f$. We infer that $T_{F} f=0$, which implies that $f=0$, since $\left.T_{F}\right|_{Y}$ is injective.

Let now $F_{-} z^{-k} F_{+}$be the Wiener-Hopf factorization of $F$ with $k=$ index $T_{F}$. One easily checks that $T_{F_{+}^{-1}} T_{F_{-}^{1} z^{k}}$ is a right inverse of $T_{F}$ when $T_{F}$ is a surjective Fredholm operator.

\section{Acknowledgements}

The authors thank the referee for careful reading the manuscript and pointing out an error in the proof of Theorem 1.

Open Access. This article is licensed under a Creative Commons Attribution 4.0 International License, which permits use, sharing, adaptation, distribution and reproduction in any medium or format, as long as you give appropriate credit 
to the original author(s) and the source, provide a link to the Creative Commons licence, and indicate if changes were made. The images or other third party material in this article are included in the article's Creative Commons licence, unless indicated otherwise in a credit line to the material. If material is not included in the article's Creative Commons licence and your intended use is not permitted by statutory regulation or exceeds the permitted use, you will need to obtain permission directly from the copyright holder. To view a copy of this licence, visit http:// creativecommons.org/licenses/by/4.0/.

Publisher's Note Springer Nature remains neutral with regard to jurisdictional claims in published maps and institutional affiliations.

\section{References}

[1] Berenstein, C.A., Gay, R.: Complex Analysis and Special Topics in Harmonic Analysis. Springer, New York (1995)

[2] Bierstedt, K.D.: An Introduction to Locally Convex Inductive Limits. Functional Analysis and Its Applications (Nice, 1986), ICPAM Lecture Notes, pp. 35-133. World Scientific Publishing, Singapore (1988)

[3] Böttcher, A., Karlovich, Y.I.: Carleson Curves, Muckenhoupt Weights, and Toeplitz. Progress in Mathematics, vol. 154. Birkhäuser Verlag, Basel (1997)

[4] Böttcher, A., Silbermann, B.: Analysis of Toeplitz Operators. Springer, Berlin (1990)

[5] Brown, A., Halmos, P.: Algebraic properties of Toeplitz operators. J. Reine Angew. Math. 231, 89-102 (1963)

[6] Conway, J.B.: Functions of One Complex Variable. Second Edition. Graduate Texts in Mathematics, p. 11. Springer, New York (1978)

[7] Doubtsov, E.: Complemented invariant subspaces and interpolation sequences. Proc. Am. Math. Soc. 135, 393-395 (2007)

[8] Domański, P.: Notes on Real Analytic Functions and Classical Operators. Topics in Complex Analysis and Operator Theory, Contemporary Mathematics, vol. 561, pp. 3-47. American Mathematical Society, Providence (2012)

[9] Domański, P., Jasiczak, M.: Toeplitz operators on the space of real analytic functions: the Fredholm property. Banach J. Math. Anal. 12, 31-67 (2018)

[10] Domański, P., Langenbruch, M.: Representation of multipliers of real analytic functions. Analysis 31, 1001-1026 (2012)

[11] Domański, P., Langenbruch, M.: Algebra of multipliers on the space of real analytic functions of one variable. Studia Math. 212, 155-171 (2012)

[12] Domański, P., Langenbruch, M.: Hadamard multipliers on spaces of real analytic functions. Adv. Math. 240, 575-612 (2013)

[13] Domański, P., Langenbruch, M.: Interpolation of holomorphic functions and surjectivity of Taylor coefficient multipliers. Adv. Math. 293, 652-674 (2016)

[14] Domański, P., Langenbruch, M.: Euler type partial differential operators on real analytic functions. J. Math. Anal. Appl. 443, 652-674 (2016)

[15] Domański, P., Langenbruch, M., Vogt, D.: Hadamard type operators on spaces of real analytic functions in several variables. J. Funct. Anal. 269, 3868-3913 (2015) 
[16] Domański, P., Langenbruch, M.: Surjectivity of Euler type differential operators on spaces of smooth functions. Trans. Amer. Math. Soc. 372, 6017-6086 (2019)

[17] Domański, P., Vogt, D.: The space of real analytic functions has no basis. Studia Math. 142, 187-200 (2000)

[18] Domański, P., Vogt, D.: Linear Topological Properties of the Space of Analytic Functions on the Real Line. Recent progress in functional analysis (Valencia, 2000), North-Holland Mathematics Studies, vol. 189, pp. 113-132. NorthHolland, Amsterdam (2001)

[19] Flajolet, P., Segdewick, R.: Mellin transforms and asymptotics: finite differences and Rice's integrals. Theor. Comput. Sci. 144, 101-124 (1995)

[20] Floret, K., Wloka, J.: Einführung in die Theorie der lokalkonvexen Räume. Lecture Notes in Mathematics, vol. 56. Springer, Berlin (1968)

[21] Gelfond, A.O.: Differenzenrechnung. Hochschulbücher für Mathematik Herausgegeben von H. Grell, K. Maruhn und W. Rinow, vol. 41. VEB Deutscher Verlag der Wissenscheften, Berlin (1958)

[22] Gohberg, I., Krupnik, N.: One-Dimensional Linear Singular Integral Equations. Volume I. Introduction. Operator Theory: Advances and Applications, vol. 53. Birkhäuser Verlag, Basel (1992)

[23] Golińska, A.: Hankel operators on the space of real analytic functions $\mathcal{A}(\mathbb{R})$. Preprint (2017)

[24] Golińska, A.: Classical operators on the space of real analytic functions. Ph.D. thesis (2019)

[25] Hörmander, L.: On the existence of real analytic solutions of partial differential equations with constant coefficients. Invent. Math. 21, 151-182 (1973)

[26] Jarchow, H.: Locally Convex Spaces. Mathematische Leitfäden. B. G. Teubner, Stuttgart (1981)

[27] Jasiczak, M.: Coburn-Simonenko theorem and invertibility of Toeplitz operators on the space of real analytic functions. J. Oper. Theory 79, 327-344 (2018)

[28] Jasiczak, M.: Semi-Fredholm Toeplitz operators on the space of real analytic functions. Accepted in Studia Math

[29] Korenblum, B., Zhu, K.: Complemented invariant subspaces in Bergman spaces. J. Lond. Math. Soc. 71, 467-480 (2005)

[30] Köthe, G.: Topological Vector Spaces. I. Die Grundlehren der mathematischen Wissenschaften, vol. 159. Springer, New York (1969)

[31] Krantz, S.G., Parks, H.R.: A Primer of Real Analytic Functions. Second Edition. Birkhäuser Advanced Texts: Basler Lehrbücher. [Birkhäuser Advanced Texts: Basel Textbooks]. Birkhäuser Boston, Inc., Boston (2002)

[32] Langenbruch, M.: Continuous linear right inverses for convolution operators in spaces of real analytic functions. Studia Math. 110, 65-82 (1994)

[33] Lindenstrauss, J., Tzafriri, L.: On the complemented subspace problem. Israel J. Math. 9, 263-269 (1971)

[34] Markushevich, A.I.: Theory of Functions of a Complex Variable. Part II. AMS Chelsea Publishing, American Mathematical Society, Providence (2005)

[35] Martineau, A.: Sur la topologie des espaces de fonctions holomorphes. Math. Ann. 163, 62-88 (1966) 
[36] Meise, R., Vogt, D.: Introduction to Functional Analysis. Oxford Graduate Texts in Mathematics, vol. 2. The Clarendon Press, New York (1997)

[37] Nikolski, N.N.: Operators, Functions, and Systems: An Easy Reading. Volume 1. Hardy, Hankel and Toeplitz. Mathematical Surveys and Monographs, vol. 92. American Mathematical Society, Providence (2010)

[38] Nörlund, N.F.: Vorlesungen über Differenzenrechnung. Chelsea, New York (1954)

[39] Pietsch, A.: Zur Theorie der $\sigma$-Transformationen in lokalkonvexen Vektorräumen. Math. Nachr. 21, 347-369 (1960)

[40] Renardy, M., Rogers, R.C.: An Introduction to Partial Differential Equations. Texts in Applied Mathematics, vol. 13. Springer, New York (1993)

[41] Roman, S.: The Umbral Calculus. Pure and Applied Mathematics, vol. 111. Academic Press, Inc., New York (1984)

[42] Trybuła, M.: Hadamard multipliers on spaces of holomorphic functions. Integral Equ. Oper. Theory 88, 249-268 (2017)

[43] Vogt, D.: Operators of Hadamard type on spaces of smooth functions. Math. Nachr. 288, 353-361 (2015)

[44] Vogt, D.: Hadamard operators on $\mathcal{D}^{\prime}\left(\mathbb{R}^{d}\right)$. Studia Math. 237, 137-152 (2017)

[45] Vogt, D.: Hadamard operators on $\mathcal{D}^{\prime}(\Omega)$. Math. Nachr. 290, 1374-1380 (2017)

[46] Vogt, D.: Surjectivity of Euler operators on temperate distributions. J. Math. Anal. Appl. 466, 1393-1399 (2018)

[47] Zhu, K.: Analysis on Fock Spaces. Springer, New York (2012)

M. Jasiczak $(\varangle)$ and A. Golińska

Faculty of Mathematics and Computer Science

Adam Mickiewicz University

ul. Uniwersytetu Poznańskiego 4

61-614 Poznań

Poland

e-mail: mjk@amu.edu.pl

Received: October 9, 2019.

Revised: January 7, 2020. 